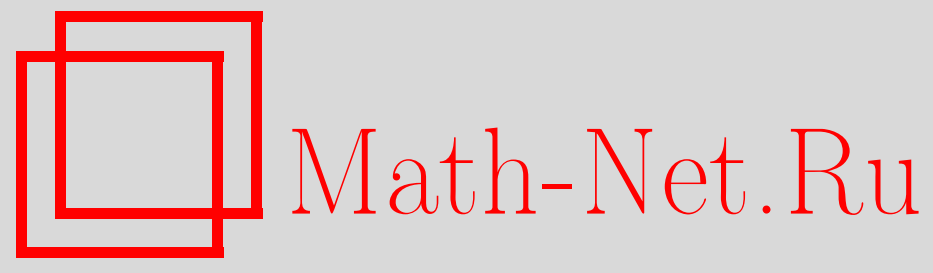

А. В. Хохлов, Анализ общих свойств кривых ползучести при циклических ступенчатых нагружениях, порождаемых линейной теорией наследственности, Вестн. Сам. гос. техн. ун-та. Сер. Физ.-мат. науки, 2017, номер 2, 326-361

DOI: https://doi.org/10.14498/vsgtu1533

Использование Общероссийского математического портала MathNet.Ru подразумевает, что вы прочитали и согласны с пользовательским соглашением

http: //www . mathnet.ru/rus/agreement

Параметры загрузки:

IP: 54.224 .187 .69

26 апреля 2023 г., 02:22:03

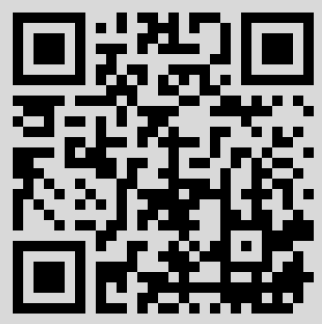


УДК 539.372

\section{Анализ общих свойств кривых ползучести при циклических ступенчатых нагружениях, порождаемых линейной теорией наследственности}

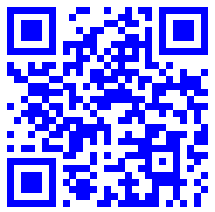

\section{А. В. Хохлов}

Московский государственный университет имени М. В. Ломоносова, Научно-исследовательский институт механики,

Россия, 119192, Москва, Мичуринский проспект, 1.

\section{Аннотация}

Аналитически изучены общие свойства кривых ползучести, порождаемых линейным определяющим соотношением вязкоупругости с произвольной функцией ползучести при циклических двухступенчатых нагружениях с произвольным коэффициентом асимметрии цикла. Исследованы интервалы монотонности и выпуклости циклических кривых ползучести, поведение последовательностей максимальных и минимальных значений деформации в каждом цикле и их полусуммы (условия их ограниченности, монотонности, сходимости), их отклонение от обычной кривой ползучести при среднем напряжении цикла, условия моделирования циклического упрочнения и разупрочнения, а также зависимость всех обнаруженных свойств от характеристик функции ползучести и параметров цикла нагружения.

Для импульсных (отнулевых), симметричных и произвольных циклических ступенчатых нагружений выведены общие формулы и точные двусторонние оценки для максимальных и минимальных значений деформации в каждом цикле и их пределов, для отклонения кривой циклической ползучести от обычной кривой ползучести при среднем напряжении цикла, для скорости накопления пластической (необратимой) деформации и рэтчетинга. Выявлена ключевая роль выпуклости вверх функции ползучести и величины предела ее производной на бесконечности; в частности, доказано, что равенство этого предела нулю является критерием отсутствия накопления пластической деформации при циклических нагружениях и критерием асимптотической симметризации отклонения деформации от кривой ползучести для среднего напряжения.

На основе сравнения обнаруженных свойств теоретических кривых с типичными свойствами экспериментальных кривых ползучести вязкоупругопластичных материалов при ступенчатых нагружениях изучены возможности линейной теории вязкоупругости по описанию различных

\section{Статья}

( () (7) Контент публикуется на условиях лицензии Creative Commons Attribution 4.0 International (https://creativecommons.org/licenses/by/4.0/deed.ru)

\section{Образец для цитирования}

Хохлов А. В. Анализ общих свойств кривых ползучести при циклических ступенчатых нагружениях, порождаемых линейной теорией наследственности // Becmн. Caм. гос. техн. ун-та. Сер. Физ.-мат. науки, 2017. Т. 21, № 2. С. 326-361. doi: 10.14498/vsgtu1533.

Сведения об авторе

Андрей Владимирович Хохлов (1) http://orcid.org/0000-0002-9212-2579

кандидат технических наук; старший научный сотрудник; лаб. упругости и пластичности; e-mail: andrey-khokhlov@ya.ru 
эффектов, наблюдаемых при циклических ступенчатых нагружениях, сферы влияния общих качественных характеристик функции ползучести и способы идентификации и настройки определяющего соотношения. В частности, доказано, что линейное соотношение вязкоупругости с произвольной функцией ползучести моделирует отсутствие рэтчетинга (и убывание последовательности модулей полусумм максимального и минимального значений деформации в каждом цикле) при симметричных циклических нагружениях, а при наложении симметричного (ступенчатого) циклического возмущения на постоянную нагрузку не происходит ускорения ползучести по сравнению с ползучестью при среднем напряжении.

Ключевые слова: линейная вязкоупругость, циклическая ползучесть, кривые ползучести при ступенчатых нагружениях, коэффициент асимметрии цикла, среднее напряжение, ускорение ползучести, пластическая деформация, рэтчетинг, циклическая стабильность.

Получение: 14 марта 2017 г. / Исправление: 17 мая 2017 г. Принятие: 12 июня 2017 г. / Публикация онлайн: 10 июля 2017 г.

Введение. Точное знание арсенала возможностей и границ применимости линейной теории вязкоупругости, имманентных свойств ее базовых теоретических кривых, вытекающих из постулатов наследственности, линейности и инвариантности интегрального оператора относительно сдвигов по времени, необходимо для грамотного моделирования и планирования экспериментов, полезно для выбора, конструирования и аттестации более сложных и точных моделей поведения реономных материалов, использующих и обобщающих линейную теорию наследственности в определенных аспектах, для совершенствования расчетных схем и методов расчета конструкций. Линейная теория играет (порой неявно) роль своеобразного «окуляра», «системы отсчета», «эталонной» сетки реперных точек, фундамента и набора инструментов для наблюдения и анализа физически и геометрически нелинейного поведения материалов и различных эффектов (их отклонений от предсказаний линейной модели как начального приближения), для построения, калибровки и сопоставления математических моделей.

Эксперименты на ползучесть при ступенчатых (с кусочно-постоянным напряжением) и циклических нагружениях - важные виды квазистатических испытаний, позволяющие обследовать разные аспекты поведения материала и уловить детали реализации многих эффектов [1-40], например, исследовать:

- зависимость кривых ползучести от уровня напряжения и предыстории нагружения, восстановление и остаточную деформацию при полной и частичной разгрузке [1-31, 36-38];

- влияние параметров начальной стадии нагружения и перестановки ступеней нагружения [2, 4, 5, 12, 29, 33-39];

- скорость затухания памяти, эффекты, сопровождающие скачок напряжения вниз или его быстрое убывание за малый промежуток времени (dip tests, rate reversal in creep tests, non-monotonic creep behavior) $[24,31,33-36]$;

- влияние скачков напряжения на длительную прочность и отклонение от правила линейного суммирования поврежденности (Miner's rule, linear 
damage rule, cumulative damage theory) [3, 4, 11, 14, 21-23, 29, 30, 40, 41];

- процессы накопления или стабилизации деформации при циклических нагружениях (cyclic softening, cyclic hardening, ratcheting, shakedown), влияние задержек напряжения, коэффициента асимметрии (коэффициента амплитуд), частоты и других параметров цикла на скорость рэтчетинга $[3,9,10,13,16,17,19,26,38,41-44]$;

- возможные проявления виброползучести (эффекты ускорения ползучести по сравнению с ползучестью при среднем или максимальном напряжении цикла, понижения деформации разрушения и долговечности) $[3,11,16,19,26,29,45-47]$ и т. п.

Результаты таких испытаний позволяют собрать более богатую информацию для выбора, идентификации и верификации определяющих соотношений по сравнению с кривыми ползучести (КП) при постоянном напряжении (которые все модели описывают адекватно при правильной настройке).

Однако для расшифровки результатов испытаний (и их планирования), правильной интерпретации, верного выбора определяющего соотношения (OC), его идентификации, настройки, грамотного применения и численной реализации необходимо точное знание общих свойств теоретических кривых ползучести при ступенчатом (и квазиступенчатом) нагружении (а такжекривых релаксации, деформирования и др.), порождаемых рассматриваемыми ОС, полученное в результате аналитического изучения их уравнений. Необходимо изучение влияния характеристик материальных функций и параметров программы нагружения (длительностей ступеней, скачков напряжения, скоростей нагружения на переходных участках, параметров цикла и частоты нагружения и т. п.) на общие качественные свойства теоретических КП (интервалы монотонности, выпуклости, точки экстремума и излома, скачки деформации и скорости деформации, асимптотику при $t \rightarrow \infty$, отклонение от обычной КП при мгновенном нагружении до характерного значения напряжения), которые порождает применяемое ОС с произволъными материальными функциями (а не только для их конкретных видов). Такое исследование не было выполнено даже для одномерного линейного интегрального ОС вязкоупругости (или ОС «нелинейной теории наследственности» Ю. Н. Работнова [4]), хотя оно весьма полезно для уточнения арсенала его возможностей и области (не)применимости и выявления индикаторов (не)линейности поведения материалов по результатам испытаний.

Данная работа - очередной этап качественного анализа линейного ОС вязкоупругости

$$
\varepsilon(t)=\int_{0}^{t} \Pi(t-\tau) d \sigma(\tau), \quad \sigma(t)=\int_{0}^{t} R(t-\tau) d \varepsilon(\tau), \quad t \geqslant 0,
$$

с произвольными функциями ползучести и релаксации $\Pi(t)$ и $R(t)$ и обобщающего его нелинейного ОС Ю. Н. Работнова $[36,37,39,48-50]$. Цель работы аналитическое изучение общих свойств КП ОС (1) при циклических ступенчатых нагружениях (их интервалов монотонности и выпуклости, поведения последовательностей максимальных и минимальных значений деформации в каждом цикле и их полусуммы, отклонения от обычной КП при среднем напряжении, скорости рэтчетинга, условий циклической стабильности и т. п.) и их зависимости от параметров цикла нагружения и характеристик функции 
ползучести.

В статье используются следующие сокращения и обозначения:

- ФР и ФП - функции релаксации и ползучести;

- КП - кривая ползучести;

- $\mathrm{h}(t)$ - функция Хевисайда;

- $\delta(t)$ - дельта-функция Дирака;

- РеМ - регулярные модели (с ФП: П $(0) \neq 0)$;

- СиМ - сингулярные модели (ФР содержит слагаемое $\eta \delta(t), \eta>0)$;

$-\hat{y}\left(t_{*}\right):=y\left(t_{*}+0\right)-y\left(t_{*}-0\right)-$ скачок функции $y(t)$ в точке $t_{*}$;

- $y(0):=y(0+)$ - предел $y(t)$ справа в точке $t=0$.

1. Некоторые свойства линейного определяющего соотношения вязкоупругости. ОС (1) описывает изотермические одноосные процессы в структурно стабильных вязкоупругопластичных материалах, характеризуемые историями напряжения $\sigma(t)$ и деформации $\varepsilon(t)$ в точке тела (входные процессы предполагаются кусочно-непрерывными и кусочно-гладкими при $t \geqslant 0)$. Операторами вида (1) часто задаются и зависимости между историями нагрузок и перемещений при испытаниях образцов на растяжение, кручение, изгиб, индентирование и т. п. Функции ползучести и релаксации $(\Phi П$ и $\Phi Р)$ в (1) предполагаются положительными и дифференцируемыми на $(0 ; \infty), \Pi(t)$ - возрастающей и выпуклой вверх на $(0 ; \infty)$ [37], а $R(t)$ - убывающей и выпуклой вниз на $(0 ; \infty)$, ФР может иметь интегрируемую особенность или $\delta$-сингулярность в точке $t=0$. Из монотонности и положительности $\Phi П$ и $\Phi Р$ на $(0 ; \infty)$ следует, что в точке $t=0$ существуют пределы справа $\Pi(0+)=\inf \Pi(t) \geqslant 0$ и $R(0+)=\sup R(t)>0(R(0+)=+\infty$, если ФР не ограничена) и предел $R(+\infty)=\inf R(t) \geqslant 0$.

Операторы (1) определены на линейном пространстве кусочно-непрерывных и кусочно-гладких функций. Они представимы в виде

$$
y(t)=y_{r}(t)+y_{s}(t)
$$

где $y_{r}(t)$ - регулярная часть (непрерывная функция, $\left.y_{r}(0)=0\right)$,

$$
y_{s}(t)=y(0) \mathrm{h}(t)+\sum_{i=1}^{n} \hat{y}\left(t_{i}\right) \mathrm{h}\left(t-t_{i}\right)
$$

- ступенчатая функция $\left(t_{i+1}>t_{i}, \hat{y}\left(t_{i}\right)\right.$ - скачок в точке $\left.t_{i}\right)$. Тогда

$$
y^{\prime}(t)=y_{r}^{\prime}(t)+y(0) \delta(t)+\sum \hat{y}\left(t_{i}\right) \delta\left(t-t_{i}\right)
$$

и оператор (1) переводит такой процесс $\sigma(t)$ в деформацию

$$
\varepsilon(t)=\int_{0}^{t} \Pi(t-\tau) \dot{\sigma}_{r}(\tau) d \tau+\sigma(0) \Pi(t)+\sum_{1}^{n} \hat{\sigma}\left(t_{i}\right) \Pi\left(t-t_{i}\right) \mathrm{h}\left(t-t_{i}\right), \quad t \geqslant 0 .
$$

Если $\Pi(0) \neq 0$ (модель регулярна), то $R(0)=1 / \Pi(0)<\infty$, и на линеале непрерывных кусочно-гладких при $t \geqslant 0$ функций операторы $(1)$ представимы в виде

$$
\begin{aligned}
& \sigma(t)=R(0) \varepsilon(t)+\int_{0}^{t} \dot{R}(t-\tau) \varepsilon(\tau) d \tau, \\
& \varepsilon(t)=\Pi(0) \sigma(t)+\int_{0}^{t} \dot{\Pi}(t-\tau) \sigma(\tau) d \tau, \quad t \geqslant 0 .
\end{aligned}
$$


Операторы (1) взаимно обратны и поэтому ФП и ФР связаны уравнением

$$
\int_{0}^{t} \Pi(t-\tau) R(\tau) d \tau=t, \text { или } \int_{0}^{t} \dot{\Pi}(t-\tau) R(\tau) d \tau+\Pi(0) R(t)=1, \quad t>0 .
$$

Зная ФР, можно найти ФП из уравнения $(3)$, и наоборот. При П $(0) \neq 0$ уравнения (3) и (2) - уравнения Вольтерры второго рода с ограниченными $($ если $\dot{\Pi}(0+)<\infty)$ ядрами, и поэтому они однозначно разрешимы в пространствах $L_{1}[0, b]$. Случай $\Pi(0)=0$ приводит к уравнению Вольтерры первого рода, некорректным задачам, нерегулярным моделям с особенностью в нуле у функции и кривых релаксации и касательного модуля диаграмм деформирования и т. п. [37, 48].

Свойства семейств основных теоретических квазистатических кривых (диаграмм деформирования при постоянных скоростях деформации или нагружения, кривых ползучести при ступенчатом нагружении, кривых ползучести и релаксации с произвольной начальной стадией нагружения и др.), порождаемых ОС (1) с произвольной ФП, необходимые математические и феноменологические ограничения на $Ф П$ и $Ф Р$ проанализированы в цикле работ $[36,37,39,48,49]$ и др. Анализ, в частности, показал, что среди моделей, описываемых ОС (1) с различными ФР и ФП, необходимо выделять три основных класса, поскольку качественные свойства базовых теоретических кривых моделей этих классов (а также особенности постановки и решения краевых задач) заметно отличаются:

1) регулярные модели $(\mathrm{PeM})$ - у которых $\Pi(0) \neq 0$ (тогда мгновенный модуль $E=R(0)=1 / \Pi(0)$ диаграмм деформирования с постоянной скоростью конечен, $\dot{\Pi}(0) / \Pi(0)=-\dot{R}(0) / R(0)$ [37], а ОС (1) и первое уравнение (3) сводятся к уравнениям Вольтерры второго рода (2) и (3));

2) сингулярные (СиМ) - модели с $\Phi$ Р, содержащей слагаемое $\eta \delta(t), \eta>0$ $(\Phi \mathrm{P} R=\eta \delta(t)$ задает ньютоновскую жидкость с ОС $\sigma=\eta \dot{\varepsilon}$ и входит слагаемым в $Ф Р$ половины реологических моделей из пружин и демпферов), тогда $\Pi(0)=0$ и $\dot{\Pi}(0)=\eta^{-1}$;

$3)$ нерегулярные модели с неограниченной $\Phi \mathrm{P}$, не содержащей слагаемого $\eta \delta(t)$, но имеющей интегрируемую особенность в точке $t=0(R(0+)=$ $=+\infty)$.

При малых временах (и больших скоростях деформации) РеМ ведут себя как твердые тела, а СиМ - как жидкости. Третий класс занимает промежуточное положение между первыми двумя. $\mathrm{K}$ нему относится, например, ФР

$$
R(t)=A t^{-u}, \quad u \in(0 ; 1), \quad A>0,
$$

задающая так называемый «фрактальный» элемент «фрактальных» моделей ("fractional models") с оператором дробного дифференцирования; соответствующая (в силу (3)) ФП имеет вид $\Pi(t)=A^{-1} C(u) t^{u}$ и обладает не только свойством П $(0)=0$, как и СиМ, но и свойством $\dot{\Pi}(0)=\infty$, переходным к $\Pi(0) \neq 0$, характеризующему РеМ.

Все структурные реологические модели, собранные из линейных пружин и демпферов посредством последовательных и параллельных соединений, описываются ОС (1). Схемы и названия всех двух-, трех- и четырехзвенных моделей (в терминологии нет единства) приведены в [37] на рис. 1. Можно 
доказать, что множество всех несократимых $n$-звенных моделей распадается ровно на два класса эквивалентности: PeM- $n$ и СиМ- $n$ (структурно различные модели мы называем эквивалентными, если они задаются одинаковыми семействами ФП или ФР). В частности:

1) эквивалентны трехзвенные РеМ Пойнтинга-Томсона и Кельвина [37, рис. 1, a];

2) все четыре РеМ-4 [37, рис. 1, в] эквивалентны модели стандартного тела (последовательному соединению моделей Максвелла и Фойгта, т. е. РеМ-2 и СиМ-2);

3) все РеМ-2k эквивалентны параллельному соединению $k$ моделей Максвелла с разными временами релаксации;

4) все СиМ-2k эквивалентны последовательному соединению $k$ моделей Фойгта с разными временами ползучести (retardation time);

5) РеМ- $(2 k+1)$ получается из СиМ- $2 k$ последовательным присоединением упругого элемента (РеM-1), а СиМ- $(2 k+1)$ - из РеM-2k параллельным подключением вязкого элемента (СиМ-1).

Например, семейство ФП

$$
\Pi(t)=\alpha t+\beta-\gamma e^{-\lambda t}, \quad \lambda>0, \quad \alpha, \beta \geqslant 0, \quad \gamma \in[0, \beta]
$$

порождает все РеМ-4 при $\gamma \in(0 ; \beta), \alpha, \beta>0$, а при $\alpha=0-$ РеМ-3 (модель Кельвина). Так как П(0) $=\beta-\gamma$, ФП (4) порождает СиМ, когда $\gamma=\beta$ : при $\lambda \beta=0$ - ньютоновскую жидкость, при $\alpha=0$ - модель Фойгта, при $\alpha>0$ получаются (все) СиМ-3. При $\gamma=0$ (4) дает модель Максвелла. Эти классические модели будут использованы для иллюстрации общих свойств циклических кривых ползучести ОС (1).

\section{2. Кривые обратной ползучести и требование выпуклости функ-} ции ползучести. Кривые ползучести (КП), порождаемые ОС (1) при нагружении $\sigma(t)=\bar{\sigma} \mathrm{h}(t)$, имеют вид

$$
\varepsilon(t, \bar{\sigma})=\bar{\sigma} \Pi(t), \quad t>0
$$

КП (5) возрастает по $t$ (так как П $(t)$ возрастает) и линейно зависит от уровня напряжений.

Отклик ОС (1) на прямоугольный импульс нагрузки

$$
\sigma(t)=\bar{\sigma}[\mathrm{h}(t)-\mathrm{h}(t-T)], \quad \bar{\sigma}>0,
$$

длительностью $T>0$, имеет вид

$$
\varepsilon(t)=\bar{\sigma} S(t ; T), \quad S(t ; T):=\Pi(t) \mathrm{h}(t)-\Pi(t-T) \mathrm{h}(t-T), \quad t \geqslant 0 .
$$

В точке $t=T \varepsilon(t)$ имеет место скачок $-\bar{\sigma} \Pi(0)$, а $\dot{\varepsilon}(t)-$ скачок $-\bar{\sigma} \dot{\Pi}(0)$. На интервале $t>T$ уравнение кривой обратной ползучести (KОП) имеет вид

$$
\varepsilon(t)=\bar{\sigma} S(t ; T), \quad S(t ; T)=\Pi(t)-\Pi(t-T), \quad t>T .
$$

У всех реономных материалов (кроме вязких жидкостей) после снятия нагрузки наблюдается постепенное убывание деформации до некоторого уровня 
$\varepsilon_{\infty} \geqslant 0$ (для сетчатых полимеров в высокоэластичном состоянии, как правило, $\left.\varepsilon_{\infty}=0\right)$. Это явление называется последействием, упругим восстановлением, обратной ползучестью [3-8, 20-29]. Из требования (нестрогого) убывания КОП (6) (с любым $T$ ) следует невозрастание Пं $(t)$ [37]. Поэтому на ФП в OC (1) следует накладьвать ограничение: П $(t)$ не имеет участков выпуклости вниз. Можно доказать, что ограничение П̈ $(t) \leqslant 0$ не является следствием остальных ограничений на $Ф Р$ и $Ф П$ (и тождества (3)): существует гладкая убывающая $\Phi$ с с $\ddot{R}(t) \geqslant 0$, такая, что соответствующая ФП строго монотонна, но имеет участок с $\ddot{\Pi}(t)>0$ (хотя на всем этом участке $\ddot{R}(t)>0)$.

Итак, $\dot{\Pi}(t)$ убывает на луче $t>0$ и $\dot{\Pi}(t)>0$, значит, существует предел $v:=\dot{\Pi}(\infty) \geqslant 0$. Так как КОП (6) убывает и положительна (ограничена снизу), она имеет при $t \rightarrow \infty$ предел $\varepsilon_{\infty} \geqslant 0$, и $\varepsilon_{\infty}=\bar{\sigma} S(\infty, T)$. Легко доказать, что $S(\infty, T)=v T$ [37], т. е. в случае $v>0$ после полной разгрузки (бесконечно долго) сохраняется остаточная деформация $\varepsilon_{\infty}=\bar{\sigma} v T$. Это означает, что при $v>0$ память интегрального оператора П, задающего ОС (1), не затухает.

Если ФП ограничена, то $v=0$ и $\varepsilon_{\infty}=0$. Нетрудно доказать, что ФП всех моделей СиМ- $2 k$ и РеM- $(2 k+1)$ ограничены, а у РeM-2k и СиМ- $(2 k+1)$, $k \in \mathbb{N}, v>0$. Может быть $v=0$ и для неограниченной $Ф П$, например, для степенной $Ф П$

$$
\Pi=c t^{u}, \quad u \in(0 ; 1), \quad c>0 .
$$

$\mathrm{У}$ классических моделей (4) $v=\alpha$ и

$$
S(t ; T)=\alpha T+c e^{-\lambda t}, \quad c:=\gamma\left(e^{\lambda T}-1\right) .
$$

В частности, $v>0$ при $\alpha>0$ (для РеМ-2 и РеM-4); у модели Максвелла и ньютоновской вязкой жидкости (СиМ-1) $\gamma=0$ и

$$
\varepsilon(t)=\bar{\sigma} \alpha T \equiv \mathrm{const}
$$

при $t>T$, т. е. упругое восстановление не происходит. У моделей Фойгта (СиМ-2) и Кельвина (РеМ-3) $\alpha=0$ и поэтому КОП (6) имеет вид

$$
\varepsilon(t)=\bar{\sigma} c e^{-\lambda t}
$$

и $\varepsilon_{\infty}=0$. Случай $\gamma<0$ в (4) приводит к нарушению ограничения П̈ $(t) \leqslant 0$, что влечет за собой возрастание кривой обратной ползучести (противоречие с экспериментальными данными).

Из ограничения П̈ $(t) \leqslant 0$ следует, что $K \Pi(5)$ всегда выпуклъ вверx, и потому $O C(1)$ не способно описъвать ускоряющуюся ползучесть (третью стадию).

Отметим, что КОП (6) с $\bar{\sigma}>0$ выпукла вниз для любъх $t>T>0$ (как в испытаниях материалов) тогда и только тогда, когда П̈( $t$ ) возрастает $(\ddot{S}(t ; T)=\ddot{\Pi}(t)-\ddot{\Pi}(t-T)>0)$. Это условие выполняется, в частности, для всех $Ф П$, представимых суммами экспонент с отрицательными показателями (моделей СиМ-2k, РеМ- $(2 k+1)$ и др.) и суммами функций $c t^{u}$.

3. Кривые ползучести при произвольных ступенчатых нагружениях. Кусочно-постоянной программе нагружения с $n$ ступенями

$$
\sigma(t)=\sum_{i=1}^{n-1} \sigma_{i}\left[\mathrm{~h}\left(t-t_{i-1}\right)-\mathrm{h}\left(t-t_{i}\right)\right]+\sigma_{n} \mathrm{~h}\left(t-t_{n-1}\right)
$$


(полагаем, что $t_{0}=0$ и $\left.t_{i}>t_{i-1}\right)$ OC (1) ставит в соответствие деформацию [36]

$$
\varepsilon(t)=\sigma_{n} \Pi\left(t-t_{n-1}\right) \mathrm{h}\left(t-t_{n-1}\right)+\sum_{i=1}^{n-1} \sigma_{i} S\left(t-t_{i-1} ; t_{i}-t_{i-1}\right), \quad t>0
$$

или

$$
\varepsilon(t)=\sigma_{1} \Pi(t)+\sum_{k=1}^{i} \hat{\sigma}_{k} \Pi\left(t-t_{k}\right), \quad t \in\left(t_{i} ; t_{i+1}\right),
$$

где $\hat{\sigma}_{i}:=\sigma_{i+1}-\sigma_{i}-$ скачки напряжения в точках $t_{i}, \sigma_{0}:=0$. Скачки $\varepsilon(t)$ и $\dot{\varepsilon}(t)$ в точках $t_{i}$ выражаются формулами [36]:

$$
\hat{\varepsilon}_{i}=\hat{\sigma}_{i} \Pi(0), \quad \hat{\dot{\varepsilon}}_{i}=\Pi(0) \hat{\dot{\sigma}}_{i}+\dot{\Pi}(0) \hat{\sigma}_{i}=\dot{\Pi}(0) \hat{\sigma}_{i} .
$$

Они не зависят от момента времени $t_{i}$ и предыстории нагружения, а только от скачка напряжения $\sigma_{i+1}-\sigma_{i}$; скачок деформации при разгрузке на $\Delta \sigma$ всегда равен по модулю скачку при догрузке на $\Delta \sigma$. Эти свойства можно использовать как индикатор применимости линейного ОС (1). Линейное ОС вязкоупругости (1) с произвольной ФП неспособно моделировать явление «дрейфа мгновенно-упругой дебормации вследствие дебормации ползучести» [51-54, 31], проявляющееся, в частности, в том, что скачок деформации при разгрузке отличается по модулю от скачка при догрузке (с тем же модулем скачка напряжения), так как зависит от предыстории нагружения. В [50] установлено, что нелинейное ОС наследственности Ю. Н. Работнова [4] способно описывать явление дрейфа.

При $t \rightarrow \infty$ из $(7)$ (в силу существования предела $S(\infty, T)=v T$ ) получим

$$
\begin{gathered}
\varepsilon(t)=\sigma_{n} \Pi\left(t-t_{n-1}\right)+p_{n-1}+o(1), \\
p_{m}:=v \sum_{i=1}^{m} \sigma_{i}\left(t_{i}-t_{i-1}\right) .
\end{gathered}
$$

Перестановка ступеней нагружения с $i<n$ не влияет на $p_{n-1}$ [39] и асимптотику (8).

Если $\sigma_{n}=0$, то $\varepsilon(t) \rightarrow p_{n-1}$ при $t \rightarrow \infty$, т. е. КП (7) имеет горизонтальную асимптоту $\varepsilon=p_{n-1}$, а $p_{n-1}$ приобретает смысл пластической (необратимой) деформации.

Если $\sigma_{n} \neq 0$, но $v=0$, то $p_{n-1}=0$ и поэтому при $t \rightarrow \infty$ имеем

$$
\varepsilon(t)-\sigma_{n} \Pi\left(t-t_{n-1}\right) \rightarrow 0, \quad \varepsilon(t)-\sigma_{n} \Pi(t) \rightarrow 0, \quad \Pi(t)-\Pi\left(t-t_{n-1}\right) \rightarrow 0 .
$$

В частности, $v=0$, если $\Pi(t)$ ограничена (например, у всех СиМ-2n и РеМ$(2 n+1))$; в этом случае $p_{n-1}=0$ и $\varepsilon(t) \rightarrow \sigma_{n} \Pi_{\infty}$ при $t \rightarrow \infty$. Если $\Pi(t)$ не ограничена и $\sigma_{n} \neq 0$, то

$$
\varepsilon(t) \sim \sigma_{n} \Pi\left(t-t_{n-1}\right),
$$

и $|\varepsilon(t)|$ неограниченно возрастает.

Если $v \neq 0$ (как у РеМ-2k и СиМ- $(2 k+1), k \in \mathbb{N})$ и $\sigma_{n} \neq 0$, то $\varepsilon(t) \rightarrow \infty$ при $t \rightarrow \infty$,

$$
\sigma_{n}\left(\Pi(t)-\Pi\left(t-t_{n-1}\right)\right) \rightarrow \sigma_{n} v t_{n-1} \neq 0
$$




$$
\varepsilon(t)-\sigma_{n} \Pi\left(t-t_{n-1}\right) \rightarrow p_{n-1}
$$

(значение $p_{n-1}$ зависит от программы $\left\{\left(t_{i}, \sigma_{i}\right)\right\}$ и может быть любым, но если все $\sigma_{i} \geqslant 0$ и хотя бы одно $\sigma_{k}>0$, то и $\left.p_{n-1}>0\right)$, а $\varepsilon(t)-\sigma_{n} \Pi(t)$, вообще говоря, не стремится к нулю при $t \rightarrow \infty$. Отметим, что если все $\sigma_{i} \geqslant 0$ для $i<n$, то отклонение $\varepsilon(t)-\sigma_{n} \Pi\left(t-t_{n-1}\right)$ убывает на луче $t>t_{n-1}$, т. к. все слагаемые в $(7)$, кроме последнего, убывают по $t$ при $t>t_{i}$.

В статье [36] изучены и проиллюстрированы общие свойства КП (7), в частности, найдены условия существования интервала убывания и точки минимума у кривых ползучести с неполной разгрузкой. В [39] исследовано влияние перестановки ступеней нагружения и обнаружено свойство асимптотической коммутативности ОС (1) при ступенчатых нагружениях. Ниже будет изучено влияние материального параметра $v$ на скорость накопления пластической деформации при циклических нагружениях.

\section{4. Деформация при циклическом импульсном нагружении с пол-} ной разгрузкой. Пусть программа нагружения состоит из одинаковых прямоугольных «отнулевых» циклов с отдыхом между ними $\left(t_{i}=i T, \sigma_{2 k-1}=\bar{\sigma}\right.$, $\left.\sigma_{2 k}=0, k=1,2, \ldots\right)$ :

$$
\sigma(t)=\bar{\sigma} \sum_{i=0}^{\infty}(-1)^{i} \mathrm{~h}\left(t-t_{i}\right), \text { или } \sigma(t)=\sum_{k=0}^{\infty} \bar{\sigma}\left[\mathrm{h}\left(t-t_{2 k}\right)-\mathrm{h}\left(t-t_{2 k+1}\right)\right] .
$$

Тогда деформация определяется по формуле

$$
\begin{aligned}
& \varepsilon(t)=\bar{\sigma} \sum_{i=0}^{\infty}(-1)^{i} \Pi\left(t-t_{i}\right) \mathrm{h}\left(t-t_{i}\right), \quad \text { или } \\
& \varepsilon(t)=\bar{\sigma} \sum_{k=0}^{\infty}\left[\Pi\left(t-t_{2 k}\right) \mathrm{h}\left(t-t_{2 k}\right)-\Pi\left(t-t_{2 k+1}\right) \mathrm{h}\left(t-t_{2 k+1}\right)\right] .
\end{aligned}
$$

Ряды (10), (11) сходятся равномерно на любом отрезке, ибо на нем отличны от нуля лишь конечное число членов ряда. Очевидно,

$$
0<\varepsilon(t) \leqslant \bar{\sigma} \Pi(t)
$$

при всех $t>0$ в силу возрастания ФП. Скачкам напряжения $\hat{\sigma}_{i}:=(-1)^{i} \bar{\sigma}$ в точках $t_{i}, i=0,1, \ldots$, соответствуют скачки $\varepsilon(t)$ и $\dot{\varepsilon}(t)$ :

$$
\hat{\varepsilon}_{i}=\hat{\sigma}_{i} \Pi(0)=(-1)^{i} \Pi(0) \bar{\sigma}, \quad \hat{\dot{\varepsilon}}_{i}=\Pi(0) \hat{\dot{\sigma}}_{i}+\dot{\Pi}(0) \hat{\sigma}_{i}=(-1)^{i} \dot{\Pi}(0) \bar{\sigma},
$$

т. к. в данном случае $\hat{\dot{\sigma}}_{i}=0$.

При $t \in\left(t_{2 i-1} ; t_{2 i}\right), i=1,2, \ldots(i-$ номер цикла) из $(11)$ имеем

$$
\varepsilon(t)=\bar{\sigma} \sum_{k=1}^{i}\left[\Pi\left(t-t_{2 k-2}\right)-\Pi\left(t-t_{2 k-1}\right)\right]=\bar{\sigma} \sum_{k=1}^{i} S\left(t-t_{2 k-2}\right),
$$

т. е. $\varepsilon(t)$ - сумма сдвигов кривой обратной ползучести (6) вдоль оси времени $\left(S(t)\right.$ - краткое обозначение для $S(t, T)$ из (6)). Каждое слагаемое $S\left(t-t_{2 k-2}\right)$ положительно (если $\Pi(t) \neq$ const) и убывает на луче $t>t_{2 k-1}$, а при $t \rightarrow \infty$ 
$S\left(t-t_{2 k-2}\right) \rightarrow v T$, где $v:=\dot{\Pi}(\infty)$. Значит, $\varepsilon(t)>0$ и монотонно убывает на $\left(t_{2 i-1} ; t_{2 i}\right)$ (лишь для упругого и вязкого элемента и модели Максвелла

$$
\varepsilon(t) \equiv \text { const }=i \alpha \bar{\sigma} T \quad \text { при } \quad t \in\left(t_{2 i-1} ; t_{2 i}\right)
$$

т. к. $S\left(t-t_{2 k-2}\right)=\alpha T$; для упругого элемента $S(t, T) \equiv 0$ при $t>T$ и $\left.\varepsilon(t) \equiv 0\right)$. Нижняя грань $\varepsilon(t)$ на интервале $\left(t_{2 i-1} ; t_{2 i}\right)$ (наименьшее значение в $i$-том цикле):

$$
\underline{\varepsilon}_{i}=\inf _{\left(t_{2 i-1} ; t_{2 i}\right)} \varepsilon(t)=\varepsilon\left(t_{2 i}-0\right)=\bar{\sigma} \sum_{k=1}^{i} S\left(t_{2 i}-t_{2 k-2}\right)=\bar{\sigma} \sum_{k=1}^{i} S(2(i-k+1) T)
$$

T. e.

$$
\underline{\varepsilon}_{i}=\bar{\sigma} \sum_{j=1}^{i} S(2 j T)
$$

Так как $S(2 j T)>0$, то $\underline{\varepsilon}_{i}>0$ (только для РеМ-1 $\underline{\varepsilon}_{i}=0$ ) и последовательность $\underline{\varepsilon}_{i}$ возрастает. Так как $S(2 j T) \geqslant S(\infty)=v T$, справедлива оценка снизу $\varepsilon_{i} \geqslant i \bar{\sigma} p$, где $p:=v T$ остаточная деформация от единичного импульса напряжения (равенство $\underline{\varepsilon}_{i}=i \bar{\sigma} p$ достигается лишь для вязкого элемента и модели Максвелла, для которых $v=\alpha$ и $S\left(t-t_{2 k-2}\right)=\alpha T=$ const).

При $t \in\left(t_{2 i-2} ; t_{2 i-1}\right), i=1, \ldots$, из $(11)$ имеем

$$
\varepsilon(t)=\bar{\sigma} \Pi(t)-\bar{\sigma} \sum_{k=1}^{i-1}\left[\Pi\left(t-t_{2 k-1}\right)-\Pi\left(t-t_{2 k}\right)\right]=\bar{\sigma} \Pi(t)-\bar{\sigma} \sum_{k=1}^{i-1} S\left(t-t_{2 k-1}\right),
$$

каждое слагаемое $S\left(t-t_{2 k-1}\right)$ положительно и убывает, следовательно, $\varepsilon(t)$ монотонно возрастает. Наибольшее значение деформации на интервале $\left(t_{2 i-2} ; t_{2 i-1}\right)$ выражается через предел в его правом конце:

$$
\begin{aligned}
\bar{\varepsilon}_{i}=\sup _{\left(t_{2 i-2} ; t_{2 i-1}\right)} \varepsilon(t)= & \varepsilon\left(t_{2 i-1}-0\right)= \\
& =\bar{\sigma} \Pi\left(t_{2 i-1}\right)-\bar{\sigma} \sum_{k=1}^{i-1}\left[\Pi\left(t_{2 i-1}-t_{2 k-1}\right)-\Pi\left(t_{2 i-1}-t_{2 k}\right)\right]
\end{aligned}
$$

T. e.

$$
\begin{aligned}
\bar{\varepsilon}_{i}=\bar{\sigma} \Pi(0)+\bar{\sigma} \sum_{j=1}^{i}[\Pi((2 j-1) T)-\Pi((2 j-2) T)] & = \\
& =\bar{\sigma} \Pi(0)+\bar{\sigma} \sum_{j=1}^{i} S((2 j-1) T) .
\end{aligned}
$$


Последовательность $\left\{\bar{\varepsilon}_{i}\right\}$ возрастает (ибо ФП возрастает и $\left.S((2 j-1) T)>0\right)$. Так как $S((2 j-1) T)>S(\infty)=v T$, справедлива оценка снизу

$$
\bar{\varepsilon}_{i} \geqslant \bar{\sigma} \Pi(0)+i \bar{\sigma} p
$$

Из (12) и (13) с учетом (6) находим

$$
\underline{\varepsilon}_{i}+\bar{\varepsilon}_{i}=\bar{\sigma} \Pi(0)+\bar{\sigma} \Pi(2 i T)-\bar{\sigma} \Pi(0)=\bar{\sigma} \Pi(2 i T),
$$

т. е. последовательность средних деформаций $\varepsilon_{i}:=0.5\left(\bar{\varepsilon}_{i}+\underline{\varepsilon}_{i}\right)$ целиком лежит на КП $\varepsilon=0.5 \bar{\sigma} \Pi(t)$ для среднего напряжения цикла $0.5 \bar{\sigma}$. Поэтому последовательность $\left\{\varepsilon_{i}\right\}$ ограничена тогда и только тогда, когда ФП ограничена. В этом случае $v=0, p=0$ и $\varepsilon_{i} \rightarrow 0.5 \bar{\sigma} \Pi(\infty)$ при $i \rightarrow \infty$.

Если же $v>0$, то $p=v T>0$, пластическая деформация (9) за $i$ циклов равна $i \bar{\sigma} p$, и

$$
\underline{\varepsilon}_{i} \geqslant i \bar{\sigma} p, \quad \bar{\varepsilon}_{i} \geqslant \bar{\sigma} \Pi(0)+i \bar{\sigma} p \quad \text { и } \quad \varepsilon_{i}=0.5 \bar{\sigma} \Pi(2 i T) \geqslant 0.5 \bar{\sigma} \Pi(0)+i \bar{\sigma} p,
$$

т. е. деформация неограниченно возрастает, пластическая деформация неограниченно накапливается со скоростъю р за иикл, и ОС моделирует рэтчетинг.

В силу формул $(12)$ и (13) размах «колебаний» $\varepsilon(t)$ на $i$-том цикле выражается формулой

$$
\begin{gathered}
\bar{\varepsilon}_{i}-\underline{\varepsilon}_{i}=\bar{\sigma} \Pi(0)+\bar{\sigma} \sum_{m=1}^{2 i}(-1)^{m+1} S(m T)=\bar{\sigma} \Pi(0)-\bar{\sigma} \sum_{k=1}^{i} \Delta(2 k T, T), \\
\Delta(t ; T)=S(t ; T)-S(t-T ; T)=\Pi(t)-2 \Pi(t-T)+\Pi(t-2 T), \quad t \geqslant 2 T .
\end{gathered}
$$

Так как ФП выпукла вверх, $\Delta(t, T) \leqslant 0$ при всех $t \geqslant 2 T$ и поэтому последовательность $\bar{\varepsilon}_{i}-\underline{\varepsilon}_{i}$ возрастает (не убывает). Лишь у модели Максвелла $($ с $\Pi=\alpha t+\beta, \alpha, \beta>0)$ и упругого и вязкого элементов (с $\alpha=0$ или $\beta=0)$ $\Delta(t, T) \equiv 0$ и поэтому $\bar{\varepsilon}_{i}-\underline{\varepsilon}_{i}=\bar{\sigma} \Pi(0)=\bar{\sigma} \beta=$ const (не зависит от $T$ ). При этом $\underline{\varepsilon}_{i}=i \bar{\sigma} \alpha T, \bar{\varepsilon}_{i}=\bar{\sigma} \beta+i \bar{\sigma} \alpha T$ и обе последовательности растут неограниченно при $\alpha>0$ (и возрастают по $T$ ).

Пример 1. Для моделей Кельвина (с ограниченной ФП П $=\beta-\gamma e^{-\lambda t}$, $\beta>\gamma)$ и Фойгта $($ с $\beta=\gamma)$

$$
\begin{gathered}
\underline{\varepsilon}_{i}=-\bar{\sigma} \sum_{k=1}^{i} \gamma\left(e^{-2 k T \lambda}-e^{-(2 k-1) T \lambda}\right)= \\
=-\bar{\sigma} \gamma\left(e^{-\lambda T}-1\right) e^{-\lambda T}\left(1-e^{-2 \lambda T i}\right)\left(1-e^{-2 \lambda T}\right)^{-1}= \\
=\bar{\sigma} \gamma\left(1-e^{-2 i \lambda T}\right)\left(e^{\lambda T}+1\right)^{-1}, \\
\bar{\varepsilon}_{i}=\bar{\sigma}(\beta-\gamma)+\bar{\sigma} \gamma\left(1-e^{-2 i \lambda T}\right)\left(1+e^{-\lambda T}\right)^{-1} .
\end{gathered}
$$

Очевидно, $\bar{\varepsilon}_{i}$ и $\underline{\varepsilon}_{i}$ связаны линейной зависимостью

$$
\bar{\varepsilon}_{i}=e^{\lambda T} \underline{\varepsilon}_{i}+\bar{\sigma}(\beta-\gamma)
$$


(для модели Фойгта $\beta-\gamma=0$ ), обе последовательности возрастают и ограничены, а при $i \rightarrow \infty$ сходятся к пределам

$$
\underline{\varepsilon}:=\bar{\sigma} \gamma\left(e^{\lambda T}+1\right)^{-1}, \quad \bar{\varepsilon}:=\bar{\sigma} \gamma\left(1+e^{-\lambda T}\right)^{-1}+\bar{\sigma}(\beta-\gamma) .
$$

Величины $\bar{\varepsilon}_{i}, \underline{\varepsilon}_{i}, \bar{\varepsilon}$ и $\underline{\varepsilon}$ зависят от отношения длительности полуцикла $T$ к времени ретардации модели $1 / \lambda$ : предел $\underline{\varepsilon}$ убывает по $T$ (по $\lambda T)$, а $\bar{\varepsilon}$ и $(\bar{\varepsilon}-\underline{\varepsilon})$ возрастают; при $T \rightarrow 0 \underline{\varepsilon} \rightarrow 0.5 \bar{\sigma} \gamma, \bar{\varepsilon} \rightarrow \bar{\sigma} \beta-0.5 \bar{\sigma} \gamma, \bar{\varepsilon}-\underline{\varepsilon} \rightarrow \bar{\sigma}(\beta-\gamma)$, а при $\lambda T \rightarrow \infty \underline{\varepsilon} \rightarrow 0, \bar{\varepsilon} \rightarrow \bar{\sigma} \beta, \bar{\varepsilon}-\underline{\varepsilon} \rightarrow \bar{\sigma} \beta$.

Модель стандартного тела (РеМ-4) получается из модели Кельвина (РеМ-3) последовательным подключением вязкого элемента $(\Pi=\alpha t)$. При последовательном соединении моделей их ФП складываются, поэтому складываются и функции (16) и последовательности $\left\{\bar{\varepsilon}_{i}\right\},\left\{\underline{\varepsilon}_{i}\right\},\left\{\bar{\varepsilon}_{i}-\underline{\varepsilon}_{i}\right\}$. Поэтому для РеM-4

$$
v=\alpha>0, \quad \bar{\varepsilon}_{i}-i \bar{\sigma} \alpha T=e^{\lambda T}\left(\underline{\varepsilon}_{i}-i \bar{\sigma} \alpha T\right)+\bar{\sigma}(\beta-\gamma),
$$

или

$$
\bar{\varepsilon}_{i}=e^{\lambda T} \underline{\varepsilon}_{i}+\left(1-e^{\lambda T}\right) \bar{\sigma} \alpha T i+\bar{\sigma}(\beta-\gamma) .
$$

Примечательно, что $\underline{\varepsilon}_{i} \rightarrow \infty$, но последовательность $\left\{\bar{\varepsilon}_{i}-\underline{\varepsilon}_{i}\right\}$ ограничена (ибо для вязкого элемента $\left.\bar{\varepsilon}_{i}-\underline{\varepsilon}_{i}=0\right)$, не зависит от $\alpha$ и совпадает с $\left\{\bar{\varepsilon}_{i}-\underline{\varepsilon}_{i}\right\}$ для PeM-3:

$$
\begin{aligned}
\bar{\varepsilon}_{i}-\underline{\varepsilon}_{i}=\bar{\sigma}(\beta-\gamma)+\bar{\sigma} \gamma(1 & \left.-e^{-2 i \lambda T}\right)\left[\left(1+e^{-\lambda T}\right)^{-1}-\left(e^{\lambda T}+1\right)^{-1}\right]= \\
& =\bar{\sigma}(\beta-\gamma)+\bar{\sigma} \gamma\left(e^{\lambda T}-1\right)\left(e^{\lambda T}+1\right)^{-1}\left(1-e^{-2 \lambda T i}\right) .
\end{aligned}
$$

При $i \rightarrow \infty \bar{\varepsilon}_{i}-\underline{\varepsilon}_{i} \rightarrow \bar{\sigma}(\beta-\gamma)+\bar{\sigma} \gamma\left(e^{\lambda T}-1\right)\left(e^{\lambda T}+1\right)^{-1}=L$; для любого $i$ $\bar{\sigma}(\beta-\gamma)<\bar{\varepsilon}_{i}-\underline{\varepsilon}_{i}<L<\bar{\sigma} \beta$; предел $L$ возрастает с ростом $\lambda T$, при $\lambda T \rightarrow 0$ $L \rightarrow \bar{\sigma}(\beta-\gamma)$, а при $\lambda T \rightarrow \infty L \rightarrow \bar{\sigma} \beta$.

Пример 2. Для модели со степенной $Ф П ~ П=c t^{u}, u \in(0 ; 1)$, имеем

$$
\begin{aligned}
& \Delta(t, T)=c t^{u}-2 c(t-T)^{u}+c(t-2 T)^{u}= \\
& \quad=c t^{u}\left[1-2\left(1-T t^{-1}\right)^{u}+\left(1-2 T t^{-1}\right)^{u}\right], \quad t \geqslant 2 T .
\end{aligned}
$$

При $t \rightarrow \infty$, очевидно, $\Delta(t, T)=-2 c u(1-u) T^{2} t^{u-2}+O\left(t^{u-3}\right)$. Последовательности

$$
\underline{\varepsilon}_{i}=\bar{\sigma} c T^{u} \sum_{k=1}^{i}\left[(2 k)^{u}-(2 k-1)^{u}\right], \quad \bar{\varepsilon}_{i}=\bar{\sigma} c T^{u} \sum_{k=1}^{i}\left[(2 k-1)^{u}-(2 k-2)^{u}\right]
$$

не ограничены (ряды с неотрицательными членами расходится по признаку сравнения); для любого $i$ функции $\bar{\varepsilon}_{i}(T), \underline{\varepsilon}_{i}(T)$ возрастают по $T$ и $\bar{\varepsilon}_{i}(0+)=0$, $\underline{\varepsilon}_{i}(0+)=0$.

На рис. $1^{1}$ приведены кривые ползучести (11) четырех моделей для нагружения с $T=5, \bar{\sigma}=1$ :

\footnotetext{
${ }^{1}$ В онлайн-версии статьи все рисунки выполнены в цвете.
} 


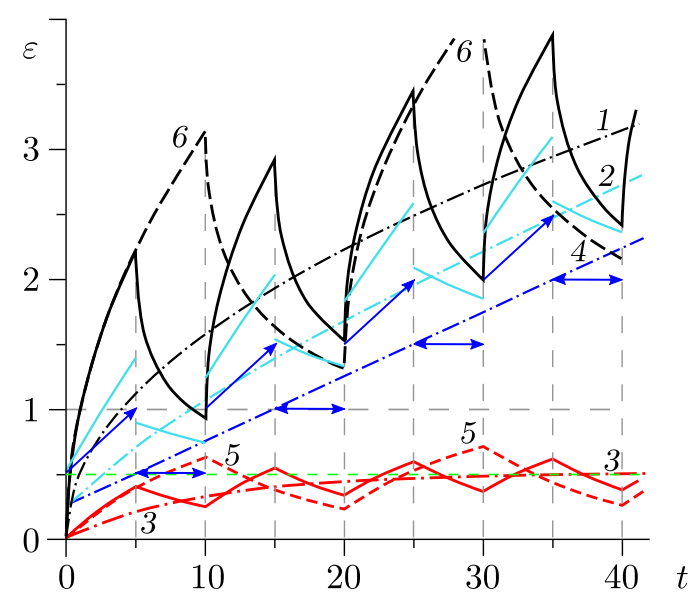

Рис. 1.

1) КП модели со степенной ФП П $=c t^{u}, c=1, u=0.5$ (черные КП);

2) КП РеМ-4 с $\Pi=\alpha t+\beta-\gamma e^{-\lambda t}, \lambda=0.1$ (время ретардации $\tau=1 / \lambda=10$ ), $\alpha=0.1, \beta=1.5, \gamma=1$ (голубые КП);

3) КП модели Фойгта с $\Pi=\gamma-\gamma e^{-\lambda t}$ с $\lambda=0.1, \gamma=1$ (красные);

4) КП модели Максвелла (синие) с $\Pi=\alpha t+\beta, \alpha=0.1, \beta=0.5$ (время релаксации $\tau=\beta / \alpha=5)$.

РеM-4 и модель Максвелла (РеМ-2) регулярны и поэтому их КП имеют в точках $t=k T$ разрывы первого рода со скачками $\pm \bar{\sigma} \Pi(0)$; свойство $v>0$ вызывает накопление пластической деформации. У модели Фойгта и степенной модели $v=0$ и $\Pi(0)=0$ и поэтому КП непрерывны при всех $t>0$. Штрихпунктирные кривые 1-4-обычные КП этих моделей при постоянном напряжении $\sigma=0.5 \bar{\sigma}$ (среднем за цикл). При достаточно больших $t$ КП РеМ4 и РеМ-2 лежат выше КП 1 степенной модели. КП вязкого элемента (не показана) не имеет разрывов и совпадает с КП модели Максвелла на интервалах $t \in\left(t_{2 i-1} ; t_{2 i}\right)$ (где КП - горизонтальные отрезки). Аналогично, КП СиМ-3 не имеют разрывов и отличаются от КП РеМ-4 только на интервалах $t \in\left(t_{2 i-2} ; t_{2 i-1}\right), i=1, \ldots, m$ (они получаются сдвигом дуги КП РеМ-4 вниз на $\beta$, а убывающие участки КП на интервалах $t \in\left(t_{2 i-2} ; t_{2 i-1}\right)$ у СиМ-3 и РеМ-4 совпадают). Для модели Фойгта и степенной модели дополнительно приведены КП с удвоенной длительностью цикла, т. е. $T=10$ (штриховые кривые 5, 6).

Исследуем условия ограниченности (существования пределов) последовательностей максимальных и минимальных деформаций (12) и (13), в частности докажем, что последовательность $\left\{\bar{\varepsilon}_{i}-\underline{\varepsilon}_{i}\right\}$ (см. (15)) ограничена для любой допустимой $Ф$ П. Будем использовать краткое обозначение $\Delta(t)$ для функции (16).

ЛЕмма 1. Пусть $T>0, \bar{\sigma}>0$, а ФП П $(t)$ дифферениируема, положительна, возрастает и выпукла вверх при $t>0$. Тогда:

1) ряд $\sum_{k=2}^{\infty} \Delta(k T)$ сходится, а его сумма выражается формулой

$$
\Sigma=v T+\Pi(0)-\Pi(T), \quad 0 \geqslant \Sigma \geqslant \Pi(0)-\Pi(T)
$$

( $\Sigma=0$ лишь для упругого и вязкого элементов и модели Максвелла); 
2) ряды $\sum_{j=1}^{\infty} \Delta((2 j+1) T)$ u $\sum_{j=1}^{\infty} \Delta(2 j T)$ сходятся, их частичные суммы связаны формулой

$$
S_{n}^{1}+S_{n}^{2}=\Pi(0)-\Pi(T)+\Pi((2 n+1) T)-\Pi(2 n T),
$$

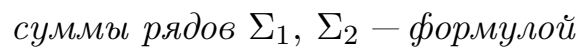

$$
\Sigma_{1}+\Sigma_{2}=v T+\Pi(0)-\Pi(T)
$$

и справедливъ неравенства $S_{n}^{2} \leqslant S_{n}^{1} \leqslant 0 u \Sigma_{2} \leqslant \Sigma_{1} \leqslant 0$.

Доказательство.

1) По формуле (16)

$$
\Delta(k T)=\Pi((k-2) T)-2 \Pi((k-1) T)+\Pi(k T),
$$

поэтому каждое слагаемое П $(k T)$ входит ровно в три члена ряда, а при их суммировании пропадает. Значит частичная сумма ряда содержит лишь две пары крайних слагаемых:

$$
S_{n}=\Pi(0)-\Pi(T)+\Pi(n T)-\Pi((n-1) T) .
$$

По теореме Лагранжа

$$
\Pi(n T)-\Pi((n-1) T)=\dot{\Pi}(\xi) T,
$$

где $\xi \in((n-1) T ; n T)$. При $n \rightarrow \infty$ имеем $\xi \rightarrow \infty, \dot{\Pi}(\xi) \rightarrow v$ (в силу убывания $\dot{\Pi}(t))$ и $S_{n} \rightarrow v T+\Pi(0)-\Pi(T)$. Оценка $\Sigma \leqslant 0$ следует из $\Delta(t, T) \leqslant 0$ при $t \geqslant 2 T$, а $\Sigma \geqslant \Pi(0)-\Pi(T)$ - из $v \geqslant 0$ (напомним, что $\dot{\Pi}(t) \geqslant 0$, и поэтому $\Pi(\infty) \geqslant 0)$.

2) Последовательность частичных сумм каждого из двух подрядов сходящегося знакопостоянного ряда $\Sigma \Delta(k T)$ ограничена, следовательно, они сходятся. По формуле (16)

$$
\Delta(2 k T)=\Pi((2 k-2) T)-2 \Pi((2 k-1) T)+\Pi(2 k T)
$$

и каждое слагаемое с четным индексом, кроме первого и последнего, входит в два члена ряда, а слагаемое с нечетным - в один. Поэтому

$$
\begin{aligned}
& S_{n}^{2}=\sum_{j=1}^{n} \Delta(2 j T)=\Pi(0)+\Pi(2 n T)+ \\
&+2 \sum_{j=1}^{n-1} \Pi(2 j T)-2 \sum_{j=1}^{n} \Pi((2 j-1) T)= \\
&=\Pi(0)+\Pi(2 n T)+2 \sum_{m=1}^{2 n-1}(-1)^{m} \Pi(m T) .
\end{aligned}
$$

Аналогично

$$
S_{n}^{1}=\sum_{j=1}^{n} \Delta((2 j+1) T)==\Pi(T)+\Pi((2 n+1) T)-2 \sum_{m=2}^{2 n}(-1)^{m} \Pi(m T),
$$

и формула (18) доказана. 
Итак, обе последовательности $\left\{\underline{\varepsilon}_{i}\right\},\left\{\bar{\varepsilon}_{i}\right\}$ возрастают, но $\left\{\bar{\varepsilon}_{i}-\underline{\varepsilon}_{i}\right\}$ ограничена (и имеет предел $\bar{\sigma} \Pi(0)-\bar{\sigma} \Sigma_{2}$ в силу леммы 1 и формулы $\left.(15)\right)$. Поэтому последовательности $\left\{\bar{\varepsilon}_{i}\right\}$ и $\left\{\underline{\varepsilon}_{i}\right\}$ могут быть ограничены только одновременно. Так как $\underline{\varepsilon}_{i}+\bar{\varepsilon}_{i}=\bar{\sigma} \Pi(2 i T)$, обе последовательности $\left\{\underline{\varepsilon}_{i}\right\},\left\{\bar{\varepsilon}_{i}\right\}$ ограничень (и сходятся) тогда и только тогда, когда ФП ограничена (в этом случае $v=0, p=0$ и $\left.\varepsilon_{i}:=0.5\left(\bar{\varepsilon}_{i}+\underline{\varepsilon}_{i}\right) \rightarrow 0.5 \bar{\sigma} \Pi(\infty)\right)$. В этом случае ряды в формулах (13) и (12) сходятся, а пределы последовательностей $\left\{\bar{\varepsilon}_{i}\right\}$ при $i \rightarrow \infty$ выражаются через их суммы $s_{1}$ и $s_{2}$ формулами

$$
\bar{\varepsilon}=\bar{\sigma} \Pi(0)+\bar{\sigma} s_{1}, \quad \underline{\varepsilon}=\bar{\sigma} s_{2} .
$$

Таким образом, доказана следующая теорема о свойствах отклика (11) на $2 T$-периодическую программу нагружения (10) из прямоугольных импульсов.

Tеорема 1. Пусть $T>0, \bar{\sigma}>0$, а ФП П $(t)$ дифферениируема, положительна, возрастает и выпукла вверх при $t>0$. Тогда кривая ползучести (11) обладает следующими свойствами:

1) на интервалах $t \in(2(i-1) T,(2 i-1) T), i=1, \ldots$, кривая ползучести (11) возрастает и выпукла вверх, а на интервалах $((2 i-1) T, 2 i T)$ $\varepsilon(t)$ убъвает (а если $\dddot{\Pi}(t) \geqslant 0$, то и въпукла вниз);

2) максимальное и минимальное значения $\bar{\varepsilon}_{i} u \underline{\varepsilon}_{i}$ деформаиии $\varepsilon(t)$ в $i$-том иикле выражаются формулами (13) и (12); $\bar{\varepsilon}_{i} \geqslant \underline{\varepsilon}_{i} \geqslant 0$, последовательности $\left\{\bar{\varepsilon}_{i}\right\}$ u $\left\{\underline{\varepsilon}_{i}\right\}$ возрастают, $\varepsilon_{i}:=0.5\left(\bar{\varepsilon}_{i}+\underline{\varepsilon}_{i}\right)=0.5 \bar{\sigma} \Pi(2 i T)$, u справедливы оценки снизу (14);

3) размах колебаний $\varepsilon(t)$ на $i$-том иикле $\bar{\varepsilon}_{i}-\underline{\varepsilon}_{i}$ выражается формулой (15), последовательность $\bar{\varepsilon}_{i}-\underline{\varepsilon}_{i}$ возрастает, ограничена и сходится; ее предел равен $\bar{\sigma} \Pi(0)-\bar{\sigma} \Sigma_{2}$;

4) последовательности $\left\{\bar{\varepsilon}_{i}\right\}$ u $\left\{\underline{\varepsilon}_{i}\right\}$ ограничены тогда и толъко тогда, когда ФП ограничена; в этом случае их пределы выражаются формулами:

$$
\bar{\varepsilon}=\bar{\sigma} \Pi(0)+\bar{\sigma} s_{1}=\bar{\sigma} \Pi(\infty)-\bar{\sigma} s_{2}, \quad \underline{\varepsilon}=\bar{\sigma} s_{2}
$$

$\left(\right.$ əде $\left.s_{1}=\sum_{j=1}^{\infty} S((2 j-1) T), s_{2}=\sum_{j=1}^{\infty} S(2 j T)\right)$

$$
\varepsilon_{i} \rightarrow 0.5 \bar{\sigma} \Pi(\infty), \quad s_{1}+s_{2}=\Pi(\infty)-\Pi(0)
$$

для деформации (11) верна двусторонняя оценка $0 \leqslant \varepsilon(t) \leqslant \bar{\varepsilon}$ при $t>0$, приращение пластической деформации за иикл $\bar{\sigma} p=\bar{\sigma} v T$ равно нулю, а ОС (1) моделирует ииклическое упрочнение материала;

5) если $v=\dot{\Pi}(\infty)>0$, то $p=v T>0$ и справедливы оценки

$$
\underline{\varepsilon}_{i} \geqslant i \bar{\sigma} p, \quad \bar{\varepsilon}_{i} \geqslant \bar{\sigma} \Pi(0)+i \bar{\sigma} p \quad u \quad \varepsilon_{i} \geqslant 0.5 \bar{\sigma} \Pi(0)+i \bar{\sigma} p
$$

в этом случае пластическая деформаиия после $i$ ииклов равна $i \bar{\sigma} р$, а ОС (1) моделирует рэтчетинг с постоянной скоростью накопления пластической деформащии б̄p за иикл (а средняя полная деформащия $\varepsilon_{i}=0.5 \bar{\sigma} \Pi(2 i T)$ pacmem eще бъстрее $)$;

6) при $T \rightarrow 0$ (для любого фиксированного $i \geqslant 1) \bar{\varepsilon}_{i} \rightarrow \bar{\sigma} \Pi(0), \underline{\varepsilon}_{i} \rightarrow 0$, а если ФП ограниченна, то $\bar{\varepsilon} \rightarrow \bar{\sigma} \Pi(0) u \underline{\varepsilon} \rightarrow 0$. 
ЗАмЕчАниЕ 1. При последовательном соединении моделей их ФП складываются, поэтому суммируются и функции $\Delta(t, T)$, и последовательности $\left\{\bar{\varepsilon}_{i}\right\}$, $\left\{\underline{\varepsilon}_{i}\right\},\left\{\bar{\varepsilon}_{i}-\underline{\varepsilon}_{i}\right\}$. Линейная комбинация (с положительными коэффициентами) $\Phi \prod$ с $v=0$ наследует свойство $v=0$; наличие хотя бы одного слагаемого с $v>0$ обеспечивает свойство $v>0$ у суммы $Ф П$.

ЗАмечание 2. С ростом $T$ величины $\bar{\varepsilon}_{i}$ и $\underline{\varepsilon}_{i}$ не обязаны всегда возрастать или убывать. Достаточные условия монотонности:

1) если функция $t \dot{\Pi}(t)$ возрастает, т. е. $t \ddot{\Pi}(t)+\dot{\Pi}(t) \geqslant 0$ (это выполняется, в частности, для степенных $\Phi П)$, то $\underline{\varepsilon}_{i}(T)$ и $\bar{\varepsilon}_{i}(T)$ возрастают, ибо производная по $T$ каждого члена $S(2 j T)=\Pi(2 j T)-\Pi((2 j-1) T)$ суммы (13) и (12) неотрицательна;

$2)$ если $t \dot{\Pi}(t)$ убывает, то $\underline{\varepsilon}_{i}(T)$ и $\bar{\varepsilon}_{i}(T)$ убывают.

$\mathrm{У}$ моделей Кельвина и Фойгта $t \dot{\Pi}(t)=\gamma \lambda t e^{-\lambda t}$ сначала возрастает, а потом убывает, $\underline{\varepsilon}_{i}(T)$ убывает, $\bar{\varepsilon}_{i}(T)$ возрастает.

5. Произвольная программа двухступенчатого циклического нагружения. В силу п. 2 теоремы 1 последовательность средних деформаций $\varepsilon_{i}$ целиком лежит на КП $\varepsilon=0.5 \bar{\sigma} \Pi(t)$ при среднем напряжении цикла $0.5 \bar{\sigma}$ (совпадает с ее значениями в точках $t=2 i T$ ). Это связано с тем, что программа импульсного нагружения (10) разлагается в сумму постоянного (среднего) напряжения $0.5 \bar{\sigma}$ и симметричного нагружения с тем же периодом и амплитудой $0.5 \bar{\sigma}$, т. е.

$$
\sigma_{0}^{+}(t, T)=0.5 \bar{\sigma}+0.5 \bar{\sigma} \sigma_{-}^{+}(t, T)
$$

и поэтому (в силу линейности ОС (1)) отклик представляется в форме

$$
\varepsilon_{0}^{+}(t, T)=0.5 \bar{\sigma} \Pi(t)+0.5 \bar{\sigma} \varepsilon_{-}^{+}(t, T)
$$

где $\varepsilon_{-}^{+}(t, T)$ - отклик на симметричное периодическое нагружение $\sigma_{-}^{+}(t, T)$ с единичной амплитудой.

Произвольная программа (равнодольного) двухступенчатого циклического нагружения

$$
\sigma(t)=\sigma_{1}+\hat{\sigma} \sum_{i=1}^{\infty}(-1)^{i} \mathrm{~h}\left(t-t_{i}\right), \quad \hat{\sigma}=\sigma_{1}-\sigma_{2},
$$

когда в моменты времени $t_{i}=i T$ ( $T$ - длительность полуцикла) происходит перескок с напряжения $\sigma_{1}$ на $\sigma_{2}$ и обратно, тоже разлагается в сумму постоянного напряжения $\sigma_{m}=0.5\left(\sigma_{1}+\sigma_{2}\right)$ и симметричного нагружения с тем же периодом $2 T$, амплитудой $\bar{\sigma}= \pm 0.5 \hat{\sigma}$ и тем же модулем скачков напряжения. В силу линейности ОС (1) отклик на нее представим в виде суммы обычной КП и отклика на симметричное циклическое нагружение $0.5 \hat{\sigma} \sigma_{-}^{+}(t, T)$ :

$$
\varepsilon(t)=\sigma_{m} \Pi(t)+0.5 \hat{\sigma} \varepsilon_{-}^{+}(t, T) .
$$

Отклик $0.5 \hat{\sigma} \varepsilon_{-}^{+}(t, T)$ равен отклонению $\varepsilon(t)$ от КП $\sigma_{m} \Pi(t)$, и анализ его свойств позволит исследовать, в частности, возможности моделирования с помощью OC (1) некоторых эффектов наложения циклического возмущения на постоянное нагружение, например, ускорения или замедления ползучести по 
сравнению с ползучестью при среднем напряжении (а не при максимальном, что принято называть виброползучестью) $[3,9,11,13,16,17,19,26,29,45-47]$. Нетрудно заметить, что, в силу линейности и инвариантности оператора (1) относительно сдвигов вдоль оси времени отклик $\varepsilon_{-}^{+}(t, T)$ выражается через линейные комбинации функции (16) и ее сдвигов по времени, и поэтому для исследования деформации в случае произвольного циклического нагружения (19) следует детально изучить свойства функции $\Delta(t, T)$.

6. Функция влияния симметричного прямоугольного импульса растяжения-сжатия. Исследуем отклик ОС (1) на трехступенчатую программу нагружения

$$
\bar{\sigma} s(t)=\bar{\sigma}[\mathrm{h}(t)-\mathrm{h}(t-T)]-\bar{\sigma}[\mathrm{h}(t-T)-\mathrm{h}(t-2 T)], \quad \bar{\sigma}>0, \quad T>0
$$

$(\sigma(t) \equiv 0$ при $t>2 T)$, состоящую из двух прямоугольных импульсов растяжения и сжатия одинаковой продолжительности и последующей полной разгрузки. Оператор (1) переводит этот процесс в

$$
\varepsilon(t)=\bar{\sigma} \Delta(t ; T)
$$

где

$$
\Delta(t ; T):=S(t ; T)-S(t-T ; T)
$$

т.e.

$$
\Delta(t ; T)=\Pi(t) \mathrm{h}(t)-2 \Pi(t-T) \mathrm{h}(t-T)+\Pi(t-2 T) \mathrm{h}(t-2 T), \quad t \geqslant 0 .
$$

Очевидно, $\Delta(t, T) \equiv 0$ при $t<0$ (и поэтому $\Delta(t-\tau, T) \mathrm{h}(t-\tau)=\Delta(t-\tau, T))$. В дальнейшем (при фиксированном $T$ ) будем использовать сокращенное обозначение $\Delta(t)$.

Если $t \in(0 ; T)$, то $\Delta(t)=\Pi(t)$ - положительная возрастающая выпуклая вверх функция.

Если $t \in(T ; 2 T)$, то $\Delta(t)=\Pi(t)-2 \Pi(t-T)=S(t ; T)-\Pi(t-T)-$ убывающая функция, причем $\Delta(2 T-0)=\Pi(2 T)-2 \Pi(T)<0$, так как $(t)$ выпукла вверх. Скачок $\Delta(t, T)$ в точке $t=T$ равен $-2 \Pi(0)$, а в точках $t=0$ и $t=2 T$ равен П $(0)$ (для сингулярных моделей П $(0)=0$ и поэтому $\Delta(t, T)$ непрерывна). Скачки производной $\Delta(t)$ в этих трех точках равны $\dot{\Pi}(0),-2 \dot{\Pi}(0)$ и $\dot{\Pi}(0)$. Если $\dddot{\Pi}(t) \geqslant 0$, то $\ddot{S}(t ; T) \geqslant 0$ и $\ddot{\Delta}(t)=\ddot{S}(t ; T)-\ddot{\Pi}(t-T) \geqslant 0$, т. е. $\Delta(t)$ выпукла вниз на интервале $(T ; 2 T)$.

Если $t \geqslant 2 T$, то $\Delta(t)$ имеет вид $(16): \Delta(t, T):=\Pi(t)-2 \Pi(t-T)+\Pi(t-2 T)$. Для удобства $\Delta(t)$ доопределена в точке $t=2 T$ по непрерывности справа: $\Delta(2 T):=\Delta(2 T+0)$. Так как

$$
2 f(t-T) \geqslant f(t)+f(t-2 T)
$$

для любой выпуклой вверх функции, $\Delta(t) \leqslant 0$ при $t \geqslant 2 T$; равенство $\Delta(t)=0$ при некотором $t>2 T$ возможно только для упругого и вязкого элементов и модели Максвелла, для которых $\Delta(t, T) \equiv 0$ при $t>2 T$. Предел $\Delta(t)$ при $t \rightarrow \infty$ всегда равен нулю, так как $\Delta(t)=S(t-T ; T)-S(t ; T)$ и существуют пределы $S(t ; T) \rightarrow v, S(t-T ; T) \rightarrow v$, где $v=\dot{\Pi}(\infty)$. Так как

$$
\dot{\Delta}(t)=\dot{\Pi}(t)-2 \dot{\Pi}(t-T)+\dot{\Pi}(t-2 T),
$$


в случае $\dddot{\Pi}(t) \geqslant 0$ будет $\dot{\Delta}(t) \geqslant 0$, т. е. $\Delta(t)$ возрастает на всем луче $t \geqslant 2 T$ (а $\mid \Delta(t \mid$ убывает).

Наибольшее значение $\Delta(t, T)$ достигается в точке $t=T$ :

$$
\sup \Delta(t)=\Delta(T-0)=\Pi(T):=\Delta_{\max } .
$$

Наименьшее значение $\Delta(t)$ достигается в точке $t=2 T$ :

$$
\inf \Delta(t)=\Delta(2 T-0)=\Pi(2 T)-2 \Pi(T):=\Delta_{\min }
$$

Если $\dot{\Pi}(t) \neq$ const, то $\Delta_{\min }<0$ и $\left|\Delta_{\min }\right|<\Delta_{\max }$, так как

$$
\Lambda_{\max }-\left|\Delta_{\min }\right|=\Pi(2 T)-\Pi(T) \quad \text { и } \quad \Pi(2 T)>\Pi(T)
$$

в силу возрастания $\Phi П\left(\left|\Delta_{\min }\right|=\Delta_{\max }\right.$ только в случае $\Pi(t)=$ const, т. е. для упругого элемента). С ростом $T \Delta_{\max }$ возрастает, а $\Delta_{\min }$ убывает:

$$
\Delta_{\min }^{\prime}(T)=2(\dot{\Pi}(2 T)-\dot{\Pi}(T)) \leqslant 0
$$

так как $\ddot{\Pi}(t) \leqslant 0$.

Таким образом, доказана следующая лемма о свойствах функции (20).

Лемма 2. Пусть $T>0, \bar{\sigma}>0, a \Phi \Pi \Pi(t)$ дифберениируема, положительна, возрастает и строго выпукла вверх при $t>0$. Тогда функиия влияния (20) обладает следующими свойствами:

1) при $t \in(0 ; T) \Delta(t)=\Pi(t)$ - положительная возрастаящая выпуклая вверх функиия;

2) при $t \in(T ; 2 T) \Delta(t)-$ убъвающая функиия и $\Delta(2 T-0)<0$;

3) на луче $t \geqslant 2 T \Delta(t)$ выражается бормулой (16), $\Delta(t)<0$ и предел $\Delta(+\infty)=0$

4) ряд $\sum_{k=2}^{\infty} \Delta(k T)$ сходится, а его сумма $\Sigma$ равна $v T+\Pi(0)-\Pi(T)$; интеграл

$$
I=\int_{2 T}^{\infty} \Delta(t) d t
$$

сходится, и для него справедлива оценка

$$
[v T+\Pi(0)-\Pi(T)] T<I<[v T+\Pi(T)-\Pi(2 T)] T
$$

5) у $\Delta(t)$ имеются ровно три точки разрьва на вещественной оси: $t=0$, $t=T$ u $t=2 T$, скачки в них равны П(0), -2П(0), П(0), а скачки производной - $\dot{\Pi}(0),-2 \dot{\Pi}(0)$ и $\dot{\Pi}(0) ; y$ нерегулярных моделей это точки устранимого разрыва и $\Delta(t)$ непрерьвна на всей оси;

6) наибольшее $u$ наименъшее значения $\Delta(t)$ достигаются в точках $t=T-0 u t=2 T-0:$

$$
\Delta_{\max }=\Delta(T-0)=\Pi(T)>0,
$$

$$
\Delta_{\min }=\Delta(2 T-0)=\Pi(2 T)-2 \Pi(T)<-\Pi(0)<0, \quad\left|\Delta_{\min }\right|<\Delta_{\max } ;
$$

$\Delta_{\max } u\left|\Delta_{\min }\right|$ возрастают с ростом $T$, a nрu $T \rightarrow 0 \Delta_{\max } \rightarrow \Pi(0), \Delta_{\min } \rightarrow-\Pi(0)$; 
7) если $\dddot{\Pi}(t) \geqslant 0$, то $\Delta(t)$ выпукла вниз на интервале $(T ; 2 T)$ и возрастает на всем луче $t \geqslant 2 T$.

ЗАмечАние 3. Пункт 4 вытекает из леммы 1 по интегральному признаку сходимости рядов.

Для моделей с ФП вида (4) (Максвелла, Фойгта, Кельвина и РеM-4)

$$
\Delta(t)=-\gamma e^{-\lambda t}+2 \gamma e^{-\lambda(t-T)}-\gamma e^{-\lambda(t-2 T)}=-\gamma\left(e^{\lambda T}-1\right)^{2} e^{-\lambda t}
$$

при $t \geqslant 2 T, \Delta(t)$ не зависит от $\alpha, \beta$ и возрастает $\left(\dddot{\Pi}(t)=\gamma \lambda^{3} e^{-\lambda t}>0\right)$,

$$
\Sigma_{1}=e^{-\lambda T} \Sigma_{2}, \quad \Sigma_{2}=-\gamma\left(e^{\lambda T}-1\right) /\left(e^{\lambda T}+1\right) .
$$

В самом деле,

$$
\begin{aligned}
\Sigma_{2}=-\gamma\left(e^{\lambda T}\right. & -1)^{2} \sum e^{-2 k \lambda T}= \\
& =-\gamma\left(e^{\lambda T}-1\right)^{2} e^{-2 \lambda T}\left(1-e^{-2 \lambda T}\right)^{-1}=-\gamma\left(e^{\lambda T}-1\right)\left(e^{\lambda T}+1\right)^{-1} .
\end{aligned}
$$

Для модели со степенной ФП П $=c t^{u}, u \in(0 ; 1)$, также $\dddot{\Pi}(t)>0$, и $\Delta(t, T)$ имеет вид (17).

На рис. 2 приведены графики функции (20) с $T=5$ для шести моделей разных классов:

1) модели со степенной ФП П $=c t^{u}, c=1, u=0.5$ (черная кривая 1) и с $c=0.5, u=0.1$ (штриховая кривая $1^{\prime}$ );

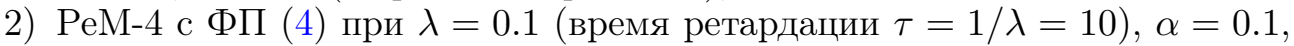
$\beta=1.5, \gamma=1$ (голубая кривая);

3) модели Фойгта с $\Pi=\gamma-\gamma e^{-\lambda t}$ при $\lambda=0.1, \gamma=1$ (красная кривая 3);

4) модели Максвелла (синяя) с $\Pi=\alpha t+\beta, \alpha=0.1, \beta=0.5$ (время релаксации $\tau=\beta / \alpha=5)$;

5) упругого элемента с $\beta=0.5$ (зеленые штриховые «ступеньки» 5);

6) вязкого элемента с $\alpha=0.1, \beta=0$ (синий «зуб»).

Нумерация кривых на рисунке совпадает с порядком перечисления моделей.

Упругий элемент, модель Максвелла и $\mathrm{PeM-4} \mathrm{регулярны}(\Pi(0) \neq 0)$, и поэтому их $\Delta(t)$ имеют ненулевые скачки в точках $t=0 ; T ; 2 T ;$ у остальных моделей $\Pi(0)=0$, и поэтому скачков нет, и $\Delta(t)$ непрерывны при всех $t \geqslant 0$. Отклик (20) для РеM-4 (и РеМ-3) на луче $t>2 T$ совпадает с откликом (20) модели Фойгта с теми же значениями $\lambda$ и $\gamma$ (так как их ФП отличаются лишь линейным слагаемым):

$$
\Delta(t)=-\gamma\left(e^{\lambda T}-1\right)^{2} e^{-\lambda t}
$$

Для близкого к нулю показателя $u$ реакция степенной модели (см. штриховую кривую $1^{\prime}$ для $\left.u=0.1\right)$ близка к реакции упругого элемента с $\Pi=c$ $(\dot{\Delta}(0)=+\infty$; свойство $\dot{\Delta}(T+0)=-\infty$ создает резкий провал, визуально не отличимый от скачка вниз, а свойства $\dot{\Delta}(2 T+0)=+\infty$ и $\Delta(t) \approx 0$ при $t>2 T$ хорошо приближают нулевой отклик упругого элемента). Для близких к единице $u$ реакция степенной модели близка к реакции вязкого элемента (нет провала при $t=T$ и $\Lambda(t) \approx 0$ на луче $t>2 T$ ). Штрихпунктиром показан 


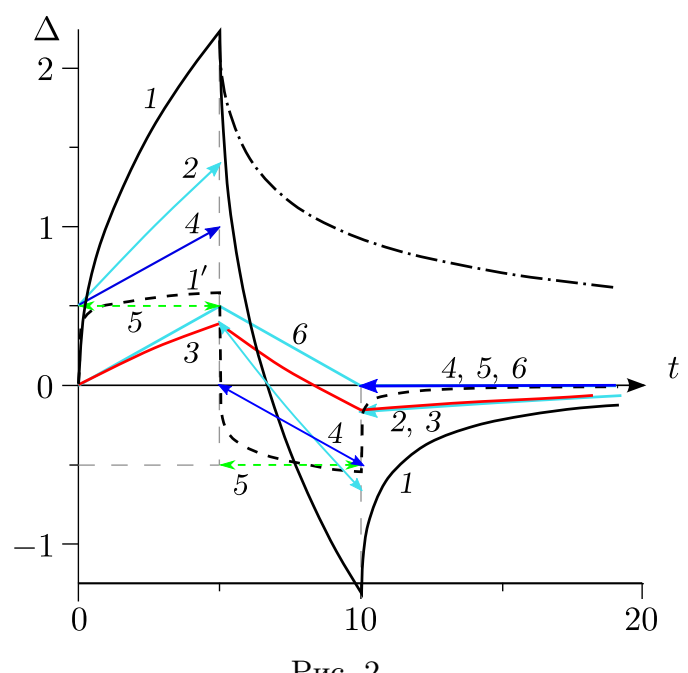

Рис. 2.

для сравнения график функции $S(t ; T)$ из (6) (т. е. кривая обратной ползучести с $\bar{\sigma}=1$ ) для степенной модели: при $t \rightarrow \infty$ она стремится к $\bar{\sigma} v T=0$, но гораздо медленнее, чем $\Delta(t)$, связанная с ней формулой $(20)$.

7. Деформация в случае симметричного циклического растяжения-сжатия. Пусть периодическая программа нагружения состоит из одинаковых прямоугольных полуциклов растяжения и сжатия длительности $T$ и амплитуды $\bar{\sigma}$ :

$$
\sigma(t)=\bar{\sigma} \mathrm{h}(t)+2 \bar{\sigma} \sum_{i=1}^{\infty}(-1)^{i} \mathrm{~h}\left(t-t_{i}\right), \text { или } \sigma(t)=\bar{\sigma} \sum_{k=0}^{\infty} s(t-2 T k),
$$

где $t_{i}=i T$. В силу линейности и инвариантности оператора (1) относительно сдвигов отклик

$$
\varepsilon(t)=\bar{\sigma} \Pi(t)+2 \bar{\sigma} \sum_{i=1}^{\infty}(-1)^{i} \Pi\left(t-t_{i}\right) \mathrm{h}\left(t-t_{i}\right)
$$

можно представить в виде суммы сдвигов функции влияния (20), изученной выше:

$$
\varepsilon(t)=\bar{\sigma} \sum_{j=0}^{\infty} \Delta(t-2 T j) \mathrm{h}(t-2 T j)=\bar{\sigma} \sum_{j=0}^{\infty} \Delta(t-2 T j) .
$$

Ряд (22) сходится при любом $t$ (равномерно на любом отрезке), ибо в точке $t$ отличны от нуля лишь конечное число членов ряда. На $i$-том периоде $t \in((2 i-2) T, 2 i T), i=1,2, \ldots$, отклик $(22)$ представляется конечной суммой c $j \leqslant i-1$ :

$$
\varepsilon(t)=\bar{\sigma} \sum_{j=0}^{i-1} \Delta(t-2 T j) \text { при } t \in((2 i-2) T, 2 i T) .
$$

При $t \in((2 i-2) T,(2 i-1) T), i=1,2, \ldots, \mathrm{K}$ (22) монотонно возрасmaem $u$ выпукла вверx, так как в представлении (22) все ненулевые слагаемые $\Delta(t-2 T j), j \leqslant i-1$, возрастают и выпуклы вверх на интервалах 
$(2 i T-2 T, 2 i T-T)$ (они совпадают с $(2 j T, 2 j T+T)$ или лежат в $(2 j T+2 T, \infty))$. Наибольшее значение деформации на интервале $\left(t_{2 i-2} ; t_{2 i-1}\right)$ (и в $i$-том цикле):

$$
\begin{aligned}
\bar{\varepsilon}_{i}=\sup _{\left(t_{2 i-2} ; t_{2 i-1}\right)} \varepsilon(t)=\varepsilon\left(t_{2 i-1}-0\right)= & \bar{\sigma} \sum_{j=0}^{i-1} \Delta((2 i-2 j-1) T-0)= \\
& =\bar{\sigma} \Delta(T-0)+\bar{\sigma} \sum_{j=0}^{i-2} \Delta((2 i-2 j-1) T)
\end{aligned}
$$

T. e.

$$
\begin{gathered}
\bar{\varepsilon}_{i}=\bar{\sigma} \Pi(T)+\bar{\sigma} \sum_{m=2}^{i} \Delta((2 m-1) T)=\bar{\sigma} \Pi(T)+\bar{\sigma} S_{i-1}^{1} \\
S_{i-1}^{1}=\sum_{k=1}^{i-1} \Delta((2 k+1) T) .
\end{gathered}
$$

Здесь $S_{i-1}^{1}, i>1,-$ частичная сумма ряда из леммы 1 . Так как $\Delta(t) \leqslant 0$ при $t \geqslant 2 T$ (см. лемму 2 ), все $\Delta((2 k+1) T) \leqslant 0, S_{i-1}^{1} \leqslant 0$ и поэтому последовательность $\left\{\bar{\varepsilon}_{i}\right\}$ убывает $\left(\bar{\varepsilon}_{i+1}-\bar{\varepsilon}_{i}=\bar{\sigma} \Delta((2 i+1) T) \leqslant 0\right)$ и $\bar{\varepsilon}_{i} \leqslant \bar{\varepsilon}_{1}=\bar{\sigma} \Pi(T)$.

Докажем, что $\bar{\varepsilon}_{i}>\bar{\sigma} \Pi(0) \geqslant 0$, а значит, последовательность $\left\{\bar{\varepsilon}_{i}\right\}$ ограничена снизу и поэтому при $i \rightarrow \infty$ имеет предел $\bar{\varepsilon}=\bar{\sigma} M(T)$, причем $\bar{\varepsilon} \geqslant \bar{\sigma} \Pi(0) \geqslant 0$.

С учетом (18) величину $\bar{\varepsilon}_{i}$ в формуле (23) можно выразить через сумму $S_{i-1}^{2}=\sum_{k=1}^{i-1} \Delta(2 k T)$ :

$\bar{\varepsilon}_{i} / \bar{\sigma}=\Pi(T)+S_{i-1}^{1}=\Pi(T)+\left[\Pi(0)-\Pi(T)+\Pi((2 i-1) T)-\Pi((2 i-2) T)-S_{i-1}^{2}\right]$, T. e.

$$
\bar{\varepsilon}_{i}=\bar{\sigma} \Pi(0)-\bar{\sigma} S_{i-1}^{2}+\bar{\sigma}[\Pi((2 i-1) T)-\Pi((2 i-2) T)] .
$$

Так как $\Pi((2 i-1) T)-\Pi((2 i-2) T)>0$ (в силу возрастания ФП) и $-S_{i-1}^{2} \geqslant 0$, величина $\bar{\varepsilon}_{i}>\bar{\sigma} \Pi(0)$. При $v \neq 0$ можно уточнить оценку по теореме Лагранжа:

$$
\Pi((2 i-1) T)-\Pi((2 i-2) T)>0=\dot{\Pi}(\xi) T,
$$

где $\xi \in((2 i-2) T ;(2 i-1) T)$. Поскольку Пं $(t)$ не возрастает, справедливы оценки

$$
\dot{\Pi}(\xi) \geqslant \dot{\Pi}((2 i-1) T) \geqslant \dot{\Pi}(+\infty)=v, \quad \Pi((2 i-1) T)-\Pi((2 i-2) T) \geqslant v T
$$

и поэтому $\bar{\varepsilon}_{i} / \bar{\sigma} \geqslant \Pi(0)+v T$ (равенство только в случае $\dot{\Pi}(t)=$ const, т. е. только для упругого и вязкого элементов и модели Максвелла). Таким образом, доказаны двусторонние оценки для последовательности $\bar{\varepsilon}_{i}$ и ее предела $\bar{\varepsilon}$ :

$$
\bar{\sigma} \Pi(T) \geqslant \bar{\varepsilon}_{i} \geqslant \bar{\sigma} \Pi(0)+\bar{\sigma} v T \geqslant 0 \text { и } \bar{\sigma} \Pi(T) \geqslant \bar{\varepsilon} \geqslant \bar{\sigma} \Pi(0)+\bar{\sigma} v T \geqslant 0 .
$$


Отметим, что для всех регулярных моделей

$$
\bar{\sigma} \Pi(0)+\bar{\sigma} v T>0
$$

а для нерегулярных с $v=0$ (например, для всех степенных ФП, для модели Фойгта и последовательных соединений любого количества моделей Фойгта)

$$
\bar{\sigma} \Pi(0)+\bar{\sigma} v T=0
$$

Из (23) и (24) следует, что предел последовательности $\left\{\bar{\varepsilon}_{i}\right\}$ при $i \rightarrow \infty$ выражается через пределы $\Sigma_{1}$ и $\Sigma_{2}$ частичных сумм $S_{i}^{1}$ и $S_{i}^{2}$ рядов из леммы 1 формулами

$$
\bar{\varepsilon}=\bar{\sigma} \Pi(T)+\bar{\sigma} \Sigma_{1}=\bar{\sigma} \Pi(0)-\bar{\sigma} \Sigma_{2}+\bar{\sigma} v T .
$$

При $t \in\left(t_{2 i-1} ; t_{2 i}\right), i=1,2, \ldots, \mathrm{KП}(22)$ убъвает и выпукла вниз (нестрого), так как в сумме $(22))$ все ненулевые слагаемые $\Delta(t-2 T j), j \leqslant i-1$, убывают и выпуклы вниз на интервалах $((2 i-1) T, 2 i T)$ (они совпадают с интервалом $(2 j T+T ; 2 j T+2 T)$, на котором функция $\Delta(t-2 T j)$ убывает и выпукла вниз). Только для упругого элемента $\varepsilon(t)=\mathrm{const}=-\bar{\sigma}$. Минимальное значение $\varepsilon(t)$ на $\left(t_{2 i-1} ; t_{2 i}\right)$ (и в $i$-том цикле) вычисляется по формуле $(22)$ :

$$
\underline{\varepsilon}_{i}=\inf _{\left(t_{2 i-1} ; t_{2 i}\right)} \varepsilon(t)=\varepsilon\left(t_{2 i}-0\right)=\bar{\sigma} \sum_{j=0}^{i-1} \Delta(2(i-j) T-0),
$$

или

$$
\underline{\varepsilon}_{i}=\bar{\sigma} \Delta(2 T-0)+\bar{\sigma} \sum_{k=2}^{i} \Delta(2 k T)=\bar{\sigma} S_{i}^{2}-\bar{\sigma} \Pi(0) .
$$

Так как $\Delta(t) \leqslant 0$ при $t \geqslant 2 T$, то

$$
\underline{\varepsilon}_{i} \leqslant \bar{\sigma} \Delta(2 T-0)=\bar{\sigma}[\Pi(2 T)-2 \Pi(T)] \leqslant-\bar{\sigma} \Pi(0) \leqslant 0, \quad i=1,2, \ldots
$$

(лишь для вязкого элемента $\left.\underline{\varepsilon}_{i}=0\right),\left|\underline{\varepsilon}_{i}\right| \geqslant \bar{\sigma} \Pi(0)$ и последовательность $\left\{\underline{\varepsilon}_{i}\right\}$ yбывает. Только для упругого и вязкого элементов и модели Максвелла, для которых $\Pi=\alpha t+\beta$ и $\Delta(t, T) \equiv 0$ при $t>2 T$, последовательность $\left\{\underline{\varepsilon}_{i}\right\}$ постоянна: $\underline{\varepsilon}_{i}=-\bar{\sigma} \Pi(0)=-\bar{\sigma} \beta$.

Чтобы доказать ограниченность последовательности $\left\{\underline{\varepsilon}_{i}\right\}$ снизу и найти оценку сверху для $\left|\varepsilon_{i}\right|$, преобразуем $(27)$, выразив $S_{i}^{2}$ через $S_{i}^{1}$ по формуле (18):

$$
\underline{\varepsilon}_{i}=-\bar{\sigma} \Pi(T)-\bar{\sigma} S_{i}^{1}+\bar{\sigma}[\Pi((2 i+1) T)-\Pi(2 i T)] .
$$

Так как $-\bar{\sigma} S_{i}^{1} \geqslant 0$ и $\Pi((2 i+1) T)-\Pi(2 i T)>0$, то $\underline{\varepsilon}_{i} \geqslant-\bar{\sigma} \Pi(T)$. При $v \neq 0$ можно уточнить эту оценку, как и при доказательстве (25): из

$$
\Pi((2 i+1) T)-\Pi(2 i T) \geqslant v T
$$

следует, что

$$
\underline{\varepsilon}_{i} \geqslant-\bar{\sigma} \Pi(T)+\bar{\sigma} v T
$$

(равенство лишь для упругого и вязкого элементов и модели Максвелла). 
Таким образом, доказаны двусторонние оценки для $\underline{\varepsilon}_{i}$ и предела $\underline{\varepsilon}$ :

$$
\begin{array}{r}
-\bar{\sigma} \Pi(T)+\bar{\sigma} v T \leqslant \underline{\varepsilon}_{i} \leqslant \bar{\sigma} \Pi(2 T)-2 \bar{\sigma} \Pi(T) \leqslant-\bar{\sigma} \Pi(0) \leqslant 0 \\
\text { и }-\bar{\sigma} \Pi(T)+\bar{\sigma} v T \leqslant \underline{\varepsilon} \leqslant-\bar{\sigma} \Pi(0) \leqslant 0 .
\end{array}
$$

В силу (23) и (28) имеем

$$
\begin{aligned}
0.5\left(\bar{\varepsilon}_{i}+\underline{\varepsilon}_{i}\right)=0.5 \bar{\sigma}[-\Delta((2 i+1) T)+\Pi((2 i+1) T)-\Pi(2 i T)]= \\
=0.5 \bar{\sigma}[\Pi(2 i T)-\Pi(2 i T-T)] .
\end{aligned}
$$

Из убывания последовательности $\left\{\underline{\varepsilon}_{i}\right\}$ и ее ограниченности снизу следует, что при $i \rightarrow \infty$ она имеет предел $\underline{\varepsilon}=\bar{\sigma} m(T)$, а из (27) и (28) следует, что он выражается формулами

$$
\underline{\varepsilon}=\bar{\sigma} \Sigma_{2}-\bar{\sigma} \Pi(0)=-\bar{\sigma} \Pi(T)-\bar{\sigma} \Sigma_{1}+\bar{\sigma} v T .
$$

Отметим, что для сходимости последовательностей $\left\{\underline{\varepsilon}_{i}\right\}$ и $\left\{\bar{\varepsilon}_{i}\right\}$ в случае симметричного цикла (21) не требуется, чтобы ФП была ограниченной, как было в случае импульсных нагружений (10) (см. п. 4). В силу формул (26), (31) и леммы 2

$$
\bar{\varepsilon}+\underline{\varepsilon}=\bar{\sigma} \Pi(T)+\bar{\sigma} \Sigma_{1}+\bar{\sigma} \Sigma_{2}-\bar{\sigma} \Pi(0)=\bar{\sigma} v T
$$

и последовательность $\bar{\varepsilon}_{i}-\left|\underline{\varepsilon}_{i}\right|=\bar{\varepsilon}_{i}+\underline{\varepsilon}_{i}$ сходится при $i \rightarrow \infty$ к числу $\bar{\sigma} v T \geqslant 0$.

Последовательность средних $\varepsilon_{i}:=0.5\left(\bar{\varepsilon}_{i}+\underline{\varepsilon}_{i}\right)$ неотрицательна и убывает (так как $\left\{\bar{\varepsilon}_{i}\right\}$ и $\left\{\underline{\varepsilon}_{i}\right\}$ убывают). Вместе с ограниченностью (сходимостью) $\left\{\bar{\varepsilon}_{i}\right\}$ и $\left\{\underline{\varepsilon}_{i}\right\}$ это означает, что при симметричном циклическом нагружении (21) рэтчетинг не происходит, деформация и петля гистерезиса стабилизируются, т. е. линейное ОС (1) верно воспроизводит эффект, наблюдаемый в испытаниях большинства реономных материалов при симметричных нагружениях.

У моделей с $v=0$ (в частности, для ограниченных $Ф П$, степенных $Ф П$, всех моделей PeM- $(2 n+1)$ и СиМ-2n)

$$
\bar{\varepsilon}=-\underline{\varepsilon}=\bar{\sigma} \Pi(0)-\bar{\sigma} \Sigma_{2}=\bar{\sigma} \Pi(T)+\bar{\sigma} \Sigma_{1}
$$

и с ростом числа циклов происходит симметризация деформации (22) относительно оси $\varepsilon=0$ :

$$
\varepsilon_{i} \rightarrow 0, \quad \bar{\varepsilon}_{i}-\left|\underline{\varepsilon}_{i}\right| \rightarrow 0 .
$$

Из $(25)$ и (29) следует, в частности, что при $T \rightarrow 0 \quad \bar{\varepsilon} \rightarrow \bar{\sigma} \Pi(0), \underline{\varepsilon} \rightarrow-\bar{\sigma} \Pi(0)$ и $\bar{\varepsilon}_{i} \rightarrow \bar{\sigma} \Pi(0), \underline{\varepsilon}_{i} \rightarrow-\bar{\sigma} \Pi(0)$ для любого фиксированного $i$, т. е. при стремлении частоты циклов к бесконечности, размах деформации уменьшается и в пределе стремится к мгновенно-упругой реакции (что и наблюдается в испытаниях реономных материалов до момента, пока не становится заметным влияние тепловыделения на механические свойства).

Подытожим обнаруженные свойства кривой ползучести (22) для произвольной периодической программы нагружения (21) с симметричным циклом.

Теорема 2. Пусть $T>0, \bar{\sigma}>0$, а функиия ползучести П $(t)$ дифберениируема, положительна, возрастает и выпукла вверх при $t>0$. Тогда: 
1) на интервалах $t \in((2 i-2) T,(2 i-1) T), i=1, \ldots$, ииклическая КП (22) возрастает и выпукла вверх, а на интервалах $((2 i-1) T, 2 i T) \varepsilon(t)$ убъвает (а если $\dddot{\Pi}(t) \geqslant 0$, то и выпукла вниз);

2) в точках $t=k T K \Pi(22)$ нерегулярных моделей имеют разрывы первого рода со скачками $2 \bar{\sigma} \Pi(0)(-1)^{k}$ и скачками скорости дебормации $\dot{\varepsilon}(t)$, равными $2 \bar{\sigma} \dot{\Pi}(0)(-1)^{k}$; КП регулярных моделей непрерывны и в точках $t=k T$, а формула для скачков $\dot{\varepsilon}(t)$ сохраняется;

3) максимальное и минимальное значения $\bar{\varepsilon}_{i} u \underline{\varepsilon}_{i}$ дебормачии $\varepsilon(t)$ в $i$ том иикле выражаются формулами (23), (24) u (27), (28); для $\bar{\varepsilon}_{i} u \underline{\varepsilon}_{i}$ справедливы двусторонние оценки (25) и (29) (не зависящие от $i$ ) $u$ $\bar{\varepsilon}_{i}-\left|\underline{\varepsilon}_{i}\right|=\bar{\varepsilon}_{i}+\underline{\varepsilon}_{i}>\bar{\sigma} v T \geqslant 0 ; \bar{\varepsilon}_{i}>\left|\underline{\varepsilon}_{k}\right|$ при любъх $i, k \geqslant 1$;

4) последовательности $\left\{\bar{\varepsilon}_{i}\right\}$ u $\left\{\underline{\varepsilon}_{i}\right\}$ убывают, ограничены и сходятся, для их пределов $\bar{\varepsilon}$ u $\underline{\varepsilon}$ справедливы бормулы (26), (31) u $\bar{\varepsilon}+\underline{\varepsilon}=\bar{\sigma} v T, a$ также двусторонние оценки (25) и (29);

5) КП (22) ограничена на луче $t \geqslant 0$ и для нее справедливы оценки

$$
\begin{gathered}
\underline{\varepsilon}=\bar{\sigma} \Sigma_{2}-\bar{\sigma} \Pi(0) \leqslant \varepsilon(t) \leqslant \bar{\varepsilon}_{1}=\bar{\sigma} \Pi(T) \text { npu } t>0, \\
\underline{\varepsilon} \leqslant \varepsilon(t) \leqslant \bar{\varepsilon}_{i+1}=\bar{\sigma} \Pi(T)+\bar{\sigma} S_{i}^{1} \text { npu } t \geqslant 2 i T
\end{gathered}
$$

6) последовательность $\varepsilon_{i}:=0.5\left(\bar{\varepsilon}_{i}+\underline{\varepsilon}_{i}\right)($ см. (30)) убывает, а при $i \rightarrow \infty$ $\varepsilon_{i} \rightarrow 0.5 \bar{\sigma} v T$

7) если ФП обладает свойством $v=0$, то $\underline{\varepsilon}=-\bar{\varepsilon}$, и с ростом числа ицилов происходит симметризаиия деформаиии (22) относительно оси $\varepsilon=0: \varepsilon_{i} \rightarrow 0 u \bar{\varepsilon}_{i}-\left|\underline{\varepsilon}_{i}\right| \rightarrow 0$

8) при $T \rightarrow 0$ для любого $i \bar{\varepsilon}_{i} \rightarrow \bar{\sigma} \Pi(0), \underline{\varepsilon}_{i} \rightarrow-\bar{\sigma} \Pi(0) u \bar{\varepsilon} \rightarrow \bar{\sigma} \Pi(0)$, $\underline{\varepsilon} \rightarrow-\bar{\sigma} \Pi(0)$.

ЗАмечание. Функции $\bar{\varepsilon}_{i}(T)$ и $\underline{\varepsilon}_{i}(T)$ не обязаны быть монотонными. Достаточные условия их монотонности:

1) если функция $t \dot{\Pi}(t)$ выпукла вверх (т. е. $2 \ddot{\Pi}(t)+t \dddot{\Pi}(t) \leqslant 0$, это выполняется, в частности, для степенных $\Phi П)$, то $\underline{\varepsilon}_{i}(T)$ и $\bar{\varepsilon}_{i}(T)$ при симметричном нагружении убывают, а размах при импульсном нагружении (15), наоборот, возрастает (ибо производная по $T$ каждого слагаемого $\Delta(2 j T)$ сумм (23), (27) и (15) отрицательна);

2) если $t \dot{\Pi}(t)$ выпукла вниз (т. е. $2 \ddot{\Pi}(t)+t \dddot{\Pi}(t) \geqslant 0$, убывает), то $\underline{\varepsilon}_{i}(T)$ и $\bar{\varepsilon}_{i}(T)$ возрастают.

У моделей Кельвина и Фойгта $t \dot{\Pi}(t)=\gamma \lambda t e^{-\lambda t}$ сначала выпукла вверх, а при больших $t$ - вниз. Поэтому при малых и больших значениях $j T \underline{\varepsilon}_{i}(T)$ и $\bar{\varepsilon}_{i}(T)$ могут вести себя по разному.

Из теоремы 2 следует, что ОС (1) с произвольной ФП моделирует отсутствие рэтчетинга при симметричных циклических нагружениях. Это и наблюдается в испытаниях структурно стабильных материалов, одинаково сопротивляющихся растяжению и сжатию $[3,7,9,10,13,17,32]$.

На рис. 3 приведены КП (22) пяти моделей (тех же, что и на рис. 2) для симметричного циклического нагружения (1) с $T=5, \bar{\sigma}=1$ :

1) КП модели со степенной ФП П $=c t^{u}, c=1, u=0.5$ (черная КП);

2) КП РеМ-4 с П $=\alpha t+\beta-\gamma e^{-\lambda t}$ с $\lambda=0.1$ (время ретардации $\tau=1 / \lambda=10$ ), $\alpha=0.1, \beta=1.5, \gamma=1$ (голубая $\mathrm{K \Pi );}$ 
3) КП модели Фойгта с $\Pi=\gamma-\gamma e^{-\lambda t}, \lambda=0.1, \gamma=1$ (красная);

4) КП модели Максвелла (синяя) с $\Pi=\alpha t+\beta, \alpha=0.1, \beta=0.5$ (время релаксации $\tau=\beta / \alpha=5, \Delta(t) \equiv 0$ при $t \geqslant 2 T, \Sigma_{1}=\Sigma_{2}=0, \bar{\varepsilon}_{i}=\Pi(T)=$ $\left.=\beta+\alpha T=\bar{\varepsilon}, \underline{\varepsilon}_{i}=-\bar{\sigma} \Pi(0)=-\bar{\sigma} \beta=\underline{\varepsilon}\right) ;$

5) КП вязкого элемента с $\Pi=\alpha t, \alpha=0.1$ (синяя ломаная: она не имеет разрывов и периодична, так как $\Delta(t) \equiv 0$ при $t \geqslant 2 T, \varepsilon(t) \geqslant 0$ на луче $\left.t \geqslant 0, \bar{\varepsilon}_{i}=\bar{\sigma} \Pi(T)=\bar{\sigma} \alpha T, \underline{\varepsilon}_{i}=0\right)$.

КП модели Максвелла и РеМ-4 имеют в точках $t_{i}=i T$ разрывы первого рода со скачками $\pm \bar{\sigma} \Pi(0)$. У модели Фойгта и степенной модели $\Pi(0)=0$ и поэтому их КП непрерывны при всех $t>0$. У этих двух моделей $v=0$ и поэтому происходит симметризация КП (22) относительно оси $\varepsilon=0$. У PeM-4, РеМ-2 и вязкого элемента (СиМ-1) $v=\alpha>0$ и поэтому $0.5\left(\bar{\varepsilon}_{i}+\underline{\varepsilon}_{i}\right) \rightarrow 0.5 \bar{\sigma} \alpha T>0$ и $\bar{\varepsilon}=|\underline{\varepsilon}|+\bar{\sigma} \alpha T>|\underline{\varepsilon}|$ (штриховые прямые на рис. $3-\varepsilon=0.5 \bar{\sigma} \alpha T=0.25$ и $\varepsilon=\bar{\sigma} \alpha T=0.5)$. Две штрихпунктирные красные кривые - КП модели Фойгта при постоянном напряжении $\bar{\sigma}= \pm 1$.

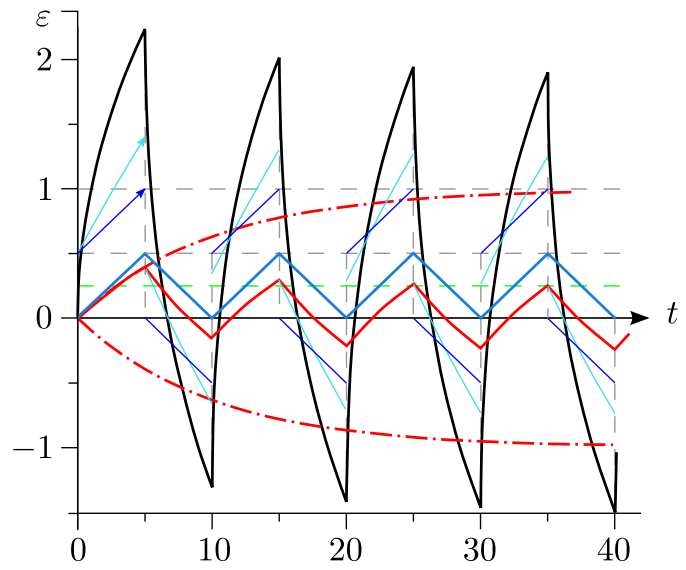

Рис. 3.

8. О кривой циклической ползучести и ее отклонении от кривой ползучести при среднем напряжении. Произвольная программа двухступенчатого циклического нагружения (19) (в моменты времени $t_{i}=i T$ происходит периодический перескок с напряжения $\sigma_{1}$ на $\sigma_{2}$ и обратно) разлагается в сумму постоянного напряжения $\sigma_{m}=0.5\left(\sigma_{1}+\sigma_{2}\right)$ и симметричного ступенчатого нагружения (21) с тем же периодом $2 T$, амплитудой $\bar{\sigma}=0.5 \hat{\sigma}$, $\hat{\sigma}=\sigma_{1}-\sigma_{2}$, и теми же скачками напряжения в точках $t_{k}=k T$, что и у (19). Отклик на нее представим в виде суммы обычной КП для среднего напряжения $\sigma_{m}$ и отклика $r(t)$ на симметричное циклическое нагружение:

$$
\begin{gathered}
\varepsilon(t)=\sigma_{m} \Pi(t)+r(t), \\
r(t)=0.5 \hat{\sigma} \sum_{i=1}^{n} \Delta(t-2 T(i-1), T), \quad t \in[0 ; 2 T n), \quad n=1,2, \ldots
\end{gathered}
$$

Свойства $r(t)$ в случае $\bar{\sigma}=0.5 \hat{\sigma}>0$ (когда $\sigma_{1}>\sigma_{2}$ ) установлены в теореме 2. Если же $\sigma_{1}<\sigma_{2}$, то $\bar{\sigma}<0$, знаки всех производных КП (22) меняются на противоположные, интервалы возрастания и убывания, выпуклости вверх и 
вниз, точки максимума и минимума меняются местами (но сохраняется их чередование). Поэтому при любых $\sigma_{1}, \sigma_{2}$ (вне зависимости от знаков $\hat{\sigma}=\sigma_{1}-\sigma_{2}$ и $\left.\sigma_{1}-\sigma_{m}\right)$ и $T>0$ для КП (32) справедлива следующая теорема.

Теорема 3. Пусть функиия ползучести П(t) дифберениируема, положительна, возрастает и выпукла вверх при $t>0$. Тогда ииклическая кривая ползучести (32) и ее отклонение $r(t)=\varepsilon(t)-\sigma_{m} \Pi(t)$ от KП для среднего напряжения $\sigma_{m}=0.5\left(\sigma_{1}+\sigma_{2}\right)$ обладают следующими свойствами:

1) бункиця $r(t)$ монотонна на интервалах $(k T, k T+T)$, интервалы возрастания и убывания чередуются; ее значения $\bar{r}_{i}:=r\left(t_{2 i-1}-0\right) u$ $\underline{r}_{i}:=r\left(t_{2 i}-0\right)$ в точках экстремума $t_{2 i-1}=(2 i-1) T$ u $t_{2 i}=2 i T$ (максимальные отклонения в $i$-том иикле) выражаются через $\Pi(t)$ бормулами (23), (24) и (27), (28) с $\bar{\sigma}=0.5 \hat{\sigma}=0.5\left(\sigma_{1}-\sigma_{2}\right)$ и заменой $\bar{\varepsilon}_{i}, \underline{\varepsilon}_{i}$ на $\bar{r}_{i}$ u $\underline{r}_{i} ;$

2) $\bar{r}_{i} \underline{r}_{i}<0$, последовательности $\bar{r}_{i}, \underline{r}_{i} u r_{i}:=0.5\left(\bar{r}_{i}+\underline{r}_{i}\right)$ монотонны, ограничены и сходятся, для их пределов $\bar{r}, \underline{r} u 0.5(\bar{r}+\underline{r})$ справедливы формуль (26), (31) $u \bar{r}+\underline{r}=\bar{\sigma} v T$, где $v=\dot{\Pi}(\infty)$;

3) $r_{i} \bar{r}_{i}>0, r_{i} \underline{r}_{i}<0$, последовательности $\left|\bar{r}_{i}\right| u\left|r_{i}\right|$ убывают, $a\left|\underline{r}_{i}\right|$ возрасmaem $u$

$$
\left|\bar{r}_{i}\right|-\left|\underline{r}_{i}\right| \geqslant|\bar{\sigma}| v T, \quad\left|r_{i}\right| \geqslant 0.5|\bar{\sigma}| v T, \quad|\bar{r}|-|\underline{r}| \geqslant|\bar{\sigma}| v T
$$

4) отклонение $r(t)$ ограничено на луче $t \geqslant 0$ :

$$
-\Pi(T)+v T-\Sigma_{1} \leqslant r(t) / \bar{\sigma} \leqslant \Pi(T),
$$

где $\Sigma_{1}$ - сумма ряда из леммы $1, \Sigma_{1} \leqslant 0$;

последовательность норм $\rho_{i}=\sup \{|r(t)| \mid t \in(2 i T ; \infty)\}$ убъвает и $\rho_{i} \rightarrow|\bar{\varepsilon}|$, zдe

$$
\bar{\varepsilon}=\bar{\sigma} \Pi(T)+\bar{\sigma} \Sigma_{1}=\bar{\sigma} \Pi(0)-\bar{\sigma} \Sigma_{2}+\bar{\sigma} v T
$$

5) полусумма экстремальных значений KП (32) выражается формулой

$$
\varepsilon_{i}=0.5 \sigma_{m}[(1+A) \Pi(2 i T)+(1-A) \Pi(2 i T-T)],
$$

где $A=\bar{\sigma} / \sigma_{m}=\left(\sigma_{1}-\sigma_{2}\right) /\left(\sigma_{1}+\sigma_{2}\right)$ - «коэфбиииент амплитуд»; последовательности отклонений

$$
\delta_{i}^{-}=\varepsilon_{i}-\sigma_{m} \Pi(2 i T-T) \quad u \quad \delta_{i}^{+}=\sigma_{m} \Pi(2 i T)-\varepsilon_{i}
$$

убъвают по модулю и сходятся $к 0.5 \sigma_{m}(1+A) v T$ и $0.5 \sigma_{m}(1-A) v T$ при $i \rightarrow \infty$; отношение

$$
\delta_{i}^{+} / \delta_{i}^{-}=(1-A) /(A+1)=\sigma_{2} / \sigma_{1}
$$

не зависит от $i, \sigma_{m} u \Pi(t)$;

6) в случае $A \in(-1 ; 1)\left(m\right.$. е. в случае $\left.\sigma_{2} / \sigma_{1}>0\right)$ для $\varepsilon_{i}$ при любом $i$ справедливы оценки

$$
\begin{gathered}
\Pi(2 i T-T) \leqslant \Pi(2 i T-T)+0.5(1+A) v T<\varepsilon_{i} / \sigma_{m}, \\
\varepsilon_{i} / \sigma_{m}<\Pi(2 i T)-0.5(1-A) v T \leqslant \Pi(2 i T)
\end{gathered}
$$


7) у моделей с $v=0$ nри $i \rightarrow \infty$ происходит асимптотическая симметризачия отклонения ииклической KП (32) от КП при среднем напряжении: $\underline{r}=-\bar{r},\left|\bar{r}_{i}\right|-\left|\underline{r}_{i}\right| \rightarrow 0, r_{i} \rightarrow 0 u \varepsilon_{i}-\sigma_{m} \Pi(2 i T) \rightarrow 0$; если $\Pi(t)$ ограничена, то $\varepsilon_{i} \rightarrow \sigma_{m} \Pi(\infty)$ и происходит симметризация ииклической КП (32) относительно прямой $\varepsilon=\sigma_{m} \Pi(\infty)$ (асимптоть $\left.K \Pi \varepsilon=\sigma_{m} \Pi(t)\right)$

8) в случае $\sigma_{m}=0$ KП (32) всегда ограничена на луче $t \geqslant 0$, а в случае $\sigma_{m} \neq 0$ KП (32) ограничена тогда и только тогда, когда ограничена $\Pi(t)$.

Таким образом, ОС (1) с произвольной ФП П $(t)$ моделирует отсутствие рэтчетинга (стабильность) при симметричных циклических нагружениях, а при наложении симметричного (ступенчатого) ииклического возмущения на постоянную нагрузку не происходит ускорения ползучести по сравнению с ползучестью при среднем напряжении. Если же есть асимметрия в циклической компоненте нагрузки (например, длительности полуциклов растяжения-сжатия не совпадают или $\left.\sigma_{-} \neq-\sigma_{+}\right)$, то при $v \neq 0$ происходит ускорение или замедление ползучести в зависимости от знака приращения пластической деформации (см. (9)) за цикл. В случае двухступенчатого цикла нагружения она выражается формулой $p=v\left(\sigma_{1} T_{1}+\sigma_{2} T_{2}\right)$, а в случае произвольного количества ступеней нагружения $\left(\sigma_{k}, T_{k}\right)$ в цикле - формулой $p=v \sum \sigma_{k} T_{k}$.

Этот аналитический результат показывает, в частности, что наблюдаемый в испытаниях различных материалов «эффект интенсификации ползучести по отношению к среднему напряжению» $[3,16,19,26,32,42]$ может быть вызван не только физическими причинами, но и неидеальностью задания (регистрации) циклической компоненты напряжения: отклонения от симметричности цикла нагружения $\left(T_{1} \neq T_{2}\right.$ или $\left.\sigma_{-} \neq-\sigma_{+}\right)$могут дать заметный (накапливающийся) вклад в наблюдаемое смещение циклической КП.

Поведение КП (32) в случае произвольного циклического нагружения (19) и ее форма на участках разгрузки (т. е участках с меньшим модулем напряжения, где слагаемые в (32) имеют разные знаки) могут существенно меняться в зависимости от знака и величины отношения

$$
A=\bar{\sigma} / \sigma_{m}=\left(\sigma_{1}-\sigma_{2}\right) /\left(\sigma_{1}+\sigma_{2}\right)
$$

амплитуды $\bar{\sigma}$ к среднему $\sigma_{m}$ (или от коэффициента асимметрии цикла $a=$ $\left.=\sigma_{2} / \sigma_{1}=(1-A) /(A+1)\right)$ и от свойств ФП. В частности, модуль $\varepsilon(t)$ не обязан убывать на участках разгрузки и может возрастать при $\sigma_{2} / \sigma_{1}>a_{+}$ или иметь точку минимума внутри интервалов $(k T,(k+1) T)$ (а не на концах), если $\sigma_{2} / \sigma_{1} \in\left(a_{-} ; a_{+}\right)$, где $0 \leqslant a_{-} \leqslant a_{+} \leqslant 1, a_{-}, a_{+}$зависят от ФП и $T$ (см. [36] и рис. 4). Значения КП (32) в концах полуциклов $\bar{\varepsilon}_{i}:=\varepsilon\left(t_{2 i-1}-0\right)$ и $\varepsilon_{i}:=\varepsilon\left(t_{2 i}-0\right)$ в общем случае уже не обязаны быть наибольшим и наименьшим значениями в $i$-том цикле. Детальный анализ общих свойств КП (32), описание возможных случаев и коррекция некоторых распространенных представлений о циклических КП - тема будущих работ автора. Здесь отметим только, что средняя деформация в цикле $\varepsilon_{i}:=0.5\left(\bar{\varepsilon}_{i}+\underline{\varepsilon}_{i}\right)$ выражается формулой

$$
\varepsilon_{i}=0.5 \sigma_{m}[(1+A) \Pi(2 i T)+(1-A) \Pi(2 i T-T)]
$$


а в случае $A \in(-1 ; 1)$ (т. е. когда $\left.a=\sigma_{2} / \sigma_{1}>0\right)$ для нее справедлива универсальная двусторонняя оценка

$$
\left|\sigma_{m}\right| \Pi(2 i T-T)<\left|\varepsilon_{i}\right|<\left|\sigma_{m}\right| \Pi(2 i T) .
$$

Отклонения величин $\varepsilon_{i}$ от значений КП при среднем напряжении выражаются формулами

$$
\begin{gathered}
\delta_{i}^{+}:=\sigma_{m} \Pi(2 i T)-\varepsilon_{i}=0.5 \sigma_{m}(1-A) S(2 i T), \\
\delta_{i}^{-}:=\varepsilon_{i}-\sigma_{m} \Pi(2 i T-T)=0.5 \sigma_{m}(1+A) S(2 i T),
\end{gathered}
$$

где $S(2 i T)=\Pi(2 i T)-\Pi(2 i T-T)>0$ - значения функции (6). Последовательности $\left|\delta_{i}^{+}\right|$и $\left|\delta_{i}^{-}\right|$убывают, так как $S(t)$ убывает (см. п. 2). Так как $S(\infty)=v T$, пределы последовательностей $\delta_{i}^{+}$и $\delta_{i}^{-}$равны $0.5 \sigma_{m}(1-A) v T$ и $0.5 \sigma_{m}(1+A) v T$ и поэтому можно уточнить оценку (34) до (33)). Лишь у моделей с $v=0 \delta_{i}^{+} \rightarrow 0$ и $\delta_{i}^{-} \rightarrow 0$ для любых $\sigma_{m}$ и $A$. С ростом $A$ модуль $\left|\delta_{i}^{+}\right|$ убывает, $\left|\delta_{i}^{-}\right|$возрастает при всех $i \geqslant 1$, а для импульсного нагружения (19) (с $\sigma_{2}=0$ или $\sigma_{1}=0$ ) $A=1$ и $\delta_{i}^{+}=0$ или $A=-1$ и $\delta_{i}^{-}=0$. Примечательно, что отношение отклонений

$$
\delta_{i}^{+} / \delta_{i}^{-}=(1-A) /(A+1)=a
$$

зависит лишь от $A$ (от $a=\sigma_{2} / \sigma_{1}$ ), но не зависит от $i, \sigma_{m}$ и функции nолзучести. Это свойство циклических КП (32), порождаемых линейным OC (1) при нагружениях вида (19), можно использовать при анализе данных испытаний как индикатор границы линейности поведения материала и (не)применимости линейного ОС (1).

На рис. 4 приведены КП (32) модели со степенной $Ф П \Pi=c t^{u}, c=0.1$, $u=0.5$, для циклических нагружений (19) с разными коэффициентами амплитуд $A=\bar{\sigma} / \sigma_{m}=\{1 ; 1 / 2 ; 1 / 3 ; 1 / 4\}$, т. е. с $\sigma_{2} / \sigma_{1}=\{0 ; 0 .(3) ; 0.5 ; 0.6\}$ (черные КП 1-4). Они показывают, что (в зависимости от величины $\sigma_{2} / \sigma_{1}$ ) циклические КП на участках разгрузки могут не только убывать, но и возрастать

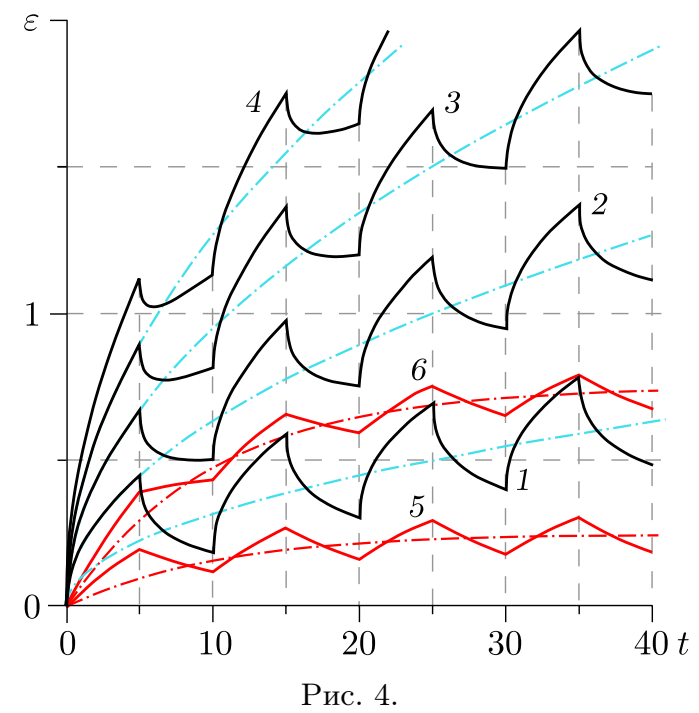


или иметь точку минимума внутри интервалов $(k T,(k+1) T)($ см. [36]), и что убывание восстанавливается на последующих циклах. Для удобства совмещения КП на одном рисунке выбрана постоянная амплитуда $\bar{\sigma}=1$ и разные средние напряжения $\sigma_{m}=\{1 ; 2 ; 3 ; 4\}$. Обычные КП для $\sigma_{m}$ показаны штрихпунктирными голубыми линиями. Красные кривые 5 и 6 - КП (32) модели Фойгта $\left(\Pi=\gamma-\gamma e^{-\lambda t}, \lambda=0.1, \gamma=0.25\right)$ для $\sigma_{m}=1$ и $\sigma_{m}=3\left(\sigma_{2} / \sigma_{1}=0 ; 0.5\right)$.

Качественно схожими свойствами обладают экспериментальные КП различных реономных материалов при ступенчатых нагружениях $[1-12,24,25$, 27-29]. Общие свойства КП, доказанные в теоремах 1-3, позволяют приглядеться к экспериментальным кривым пристальнее и попытаться разглядеть дополнительные детали, на которых акцентируют внимание теоремы (например, с целью адекватного выбора типа и характеристик функции ползучести или поиска признаков, свидетельствующих об отклонении от линейности в поведении материала, или с целью поверки надежности регистрации и обработки данных измерений).

9. Заключение. В статье продолжен качественный анализ линейного определяющего соотношения (ОС) вязкоупругости (1) с произвольной (возрастающей выпуклой вверх) функцией ползучести. Аналитически изучены общие свойства и качественные особенности кривых ползучести (32), порождаемых ОС (1) при циклических двухступенчатых нагружениях (19) с произвольным коэффициентом асимметрии цикла. Для импульсных, симметричных и произвольных циклических ступенчатых нагружений получены общие формулы и точные двусторонние оценки для максимальных и минимальных значений деформации в каждом цикле и их пределов, для отклонения кривой циклической ползучести от обычной кривой ползучести при среднем напряжении цикла, для скорости накопления пластической (необратимой) деформации и рэтчетинга. Исследованы интервалы монотонности и выпуклости кривых циклической ползучести, поведение последовательностей максимальных и минимальных значений деформации и их полусуммы (условия их ограниченности, монотонности, сходимости), возможности моделирования циклического упрочнения и разупрочнения, а также зависимость всех обнаруженных свойств от характеристик функции ползучести и параметров цикла нагружения. Основные свойства кривых циклической ползучести ОС (1) собраны в теоремах 1-3 (пп. 4, 7, 8 статьи). В частности, выявлены ключевые роли выпуклости вверх функции ползучести и величины предела ее производной $v=\dot{\Pi}(\infty)$ : доказано, что равенство $v$ нулю является критерием полного восстановления после снятия (произвольной) нагрузки и затухания памяти ОС (1), критерием отсутствия накопления пластической деформации при циклической ползучести и критерием асимптотической симметризации кривых циклической ползучести относительно КП при среднем напряжении.

Сравнение обнаруженных свойств теоретических кривых ползучести (32) с типичными свойствами экспериментальных кривых ползучести вязкоупругопластичных материалов при ступенчатых нагружениях позволило исследовать возможности ОС (1) по описанию различных эффектов при циклических ступенчатых нагружениях (в частности, выявлен ряд эффектов, которые линейное $\mathrm{OC}$ (1) не способно описывать), сферы влияния качественных характеристик функции ползучести и способы идентификации и настройки ОС. В частности, доказано, что ОС (1) с произвольной ФП моделирует 
отсутствие рэтчетинга (стабильность) при симметричных циклических нагружениях, а при наложении симметричного (ступенчатого) циклического возмущения на постоянную нагрузку не происходит ускорения ползучести по сравнению с ползучестью при среднем напряжении.

Точное знание арсенала возможностей и границ применимости линейной теории вязкоупругости, свойств ее базовых теоретических кривых, вытекающих из постулатов наследственности, линейности и инвариантности интегрального оператора (1) относительно сдвигов по времени, необходимо для грамотного моделирования и планирования экспериментов, полезно для выбора, конструирования и аттестации более сложных и точных моделей поведения реономных материалов, обобщающих линейную теорию в определенных аспектах, для совершенствования расчетных схем и методов расчета конструкций.

Конкурирующие интересы. У меня нет конкурирующих интересов.

Авторская ответственность. Я несу полную ответственность за предоставление окончательной версии рукописи в печать. Окончательная версия рукописи мною одобрена.

Финансирование. Работа выполнена при поддержке РФФИ (грант № 17-08-01146_а).

\section{Библиографический список}

1. Качанов Л. М. Теория ползучести. М.: Физматгиз, 1960. 456 с.

2. Наместников В. С., Хвостунков А. А. Ползучесть дюралюмина при постоянных и переменных нагрузках // ПМТФ, 1960. Т. 1, № 4. С. 90-95.

3. Kennedy A. J. Processes of creep and fatigue in metals / Wiley series on the science and technology of materials. vol. 19. New York: Wiley, 1963. 480 pp.

4. Работнов Ю. Н. Ползучесть элементов конструкиий. М.: Наука, 1966. 752 с.

5. Odquist F. K. G. Mathematical Theory of Creep and Creep Rupture. Oxford: Clarendon ress, 1966. $170 \mathrm{pp}$.

6. Самарин Ю. П., Сорокин О. В. О ползучести поливинилхлоридного пластиката при переменных нагрузках // Докл. АН СССР, 1970. Т. 195, № 2. С. 333-336.

7. Бугаков И. И. Ползучесть полимерных материалов. М.: Наука, 1973. 287 с.

8. Findley W. N., Lai J. S., Onaran K. Creep and Relaxation of Nonlinear Viscoelastic aterials. Amsterdam: North Holland, 1976. 368 pp.

9. Стрижало В. А. Циклическая прочность и ползучесть металлов при малочикловом нагружении в условиях низких и высоких температур. Киев: Наукова думка, 1978. 238 c.

10. Kujawski D., Kallianpur V., Krempl E. An experimental study of uniaxial creep, cyclic creep and relaxation of aisi type 304 stainless steel at room temperature // J. Mech. Phys. Solids, 1980. vol. 28, no. 2. pp. 129-148. doi : 10.1016/0022-5096(80)90018-6.

11. Шестериков С. А., Локощенко А. М. Ползучесть и длительная прочность металлов / Итоги науки и техники. Сер. Механ. деформ. тверд. тела, Т. 13. М.: ВИНИТИ, 1980. C. $3-104$.

12. Малинин Н. Н. Расчеты на ползучесть элементов машиностроительных конструкиий. М.: Машиностроение, 1981. 221 с.

13. Москвитин В. В. Циклическое нагружение элементов конструкиий. М.: Наука, 1981. $344 \mathrm{c}$.

14. Локощенко А. М., Наместникова И. В., Шестериков С. А. Описание длительной прочности при ступенчатом изменении напряжения // Проблемъ прочности, 1981. № 10. C. $47-51$. 
15. Cho U. W., Findley W. N. Creep and Plastic Strains of 304 Stainless Steel at $593^{\circ} \mathrm{C}$ Under Step Stress Changes, Considering Aging // J. Appl. Mech., 1982. vol. 49, no. 2. pp. 297-304. doi : 10.1115/1.3162084.

16. Голуб В. П. Циклическая ползучесть жаропрочных никелевых сплавов. Киев: Наумова думка, 1983. 224 с.

17. Гохфельд Д. А., Садаков О. С. Пластичность и ползучесть элементов конструкиий при повторных нагружениях. М.: Машиностроение, 1984. 256 с.

18. Малинин Н Н. Ползучесть в обработке металлов давлением. М.: Машиностроение, 1986. 221 c.

19. Голуб В. П. Исследования в области циклической ползучести материалов (обзор) // Прикладная механика, 1987. Т. 23, № 12. С. 3-19.

20. Tschoegl N. W. The Phenomenological Theory of Linear Viscoelastic Behavior. Berlin: Springer, 1989. 769 pp.

21. Никитенко А. Ф. Ползучесть и длительная прочность металлических материалов. Новосибирск: НГАСУ, 1997. 278 с.

22. Локощенко А. М. Ползучесть и длительная прочность металлов в агрессивных средаx. М.: МГУ, 2000. 179 c.

23. Радченко В. П., Саушкин М. Н. Ползучесть и релаксаиия остаточных напряжений в упрочнённых конструкциях. М.: Машиностроение-1, 2005. 226 с.

24. Hamouda B. H., Laiarinandrasana L., Piques R. Viscoplastic behavior of a medium density polyethylene (MDPE): constitutive equations based on double nonlinear deformation model // Int. J. Plasticity, 2007. vol.23, no. 8. pp. 1307-1327. doi: 10.1016/j.ijplas. 2006. 11.007.

25. Betten J. Creep Mechanics. Berlin, Heidelberg: Springer-Verlag, 2008. 267 pp.

26. Радченко В. П., Кичаев П. Е. Энергетическая концепчия ползучести и виброползучести металлов. Самара: Самар. гос. тех. ун-т, 2011. 157 с.

27. Darabi M. K, Al-Rub R. K. A., Masad E. A., Huang C.-W., Little D. N. A modified viscoplastic model to predict the permanent deformation of asphaltic materials under cycliccompression loading at high temperatures // Int. J. Plasticity, 2012. vol. 35. pp. 100-134. doi: 10.1016/j.ijplas.2012.03.001.

28. Bergstrom J. S. Mechanics of Solid Polymers. Theory and Computational Modeling. Elsevier: William Andrew, 2015. 520 pp.

29. Локощенко А. М. Ползучесть и длительная прочность металлов. М.: Физматлит, 2016. $504 \mathrm{c}$.

30. Локощенко А. М., Фомин Л. В. Длительное разрушение пластин при переменных изгибающих моментах в присутствии агрессивной среды // Прикл. матем. и механ., 2016. T. 80 , № 2. C. $276-284$.

31. Dandrea J., Lakes R. S. Creep and creep recovery of cast aluminum alloys // Mech. TimeDepend. Mater., 2009. vol.13, no. 4. pp. 303-315. doi:10.1007/s11043-009-9089-6.

32. Taleb L., Cailletaud G. Cyclic accumulation of the inelastic strain in the 304L SS under stress control at room temperature: Ratcheting or creep? // Int. J. Plasticity, 2011. vol. 27, no. 12. pp. 1936-1958. doi: 10.1016/j.ijplas.2011.02.001.

33. Khan F., Yeakle C. Experimental investigation and modeling of non-monotonic creep behavior in polymers // Int. J. Plasticity, 2011. vol.27, no.4. pp. 512-521. doi: 10.1016/j. ijplas.2010.06.007.

34. Drozdov A. D. Time-dependent response of polypropylene after strain reversal// Int. J. of Solids and Structures, 2010. vol. 47, no. 24. pp. 3221-3233. doi : 10.1016/j.ijsolstr. 2010. 08.001.

35. Drozdov A. D., Dusunceli N. Unusual mechanical response of carbon black-filled thermoplastic elastomers // Mechanics of Materials, 2014. vol.69, no. 1. pp. 116-131. doi: 10.1016/ j.mechmat . 2013.09.019. 
36. Хохлов А. В. Свойства семейств кривых ползучести при ступенчатом нагружении линейного определяющего соотношения вязкоупругости // Проблемы прочности и пластичности, 2015. Т. 77, № 4. С. 344-359.

37. Хохлов А. В. Качественный анализ общих свойств теоретических кривых линейного определяющего соотношения вязкоупругости // Наука и образование, 2016. № 5. С. 187245, http://technomag.bmstu.ru/doc/840650.html.

38. Хохлов А. В. Нелинейная модель вязкоупругопластичности типа Максвелла: свойства кривых ползучести при ступенчатых нагружениях и условия накопления пластической деформации // Машиностроение и инженерное образование, 2016. № 3. С. 55-68.

39. Хохлов А. В. Асимптотическая коммутативность кривых ползучести при ступенчатом нагружении в линейной теории наследственности // Машиностроение и инженерное образование, 2016. №1. С. 70-82.

40. Хохлов А. В. Кривые длительной прочности нелинейной модели вязкоупругопластичности типа Максвелла и правило суммирования поврежденности при ступенчатых нагружениях // Вестн. Сам. гос. техн. ун-та. Сер. Физ.-мат. науки, 2016. Т. 20, № 3. C. 524-543. doi: 10.14498/vsgtu1512.

41. Fatemi A., Yang L. Cumulative fatigue damage and life prediction theories: A survey of the state of the art for homogeneous materials // Int. J. Fatigue, 1998. vol. 20, no.1. pp. 9-34. doi : 10.1016/S0142-1123(97)00081-9.

42. Голуб В. П. О некоторых эффектах ползучести при циклических нагружениях // Проблемы прочности, 1987. № 5. С. 20-24.

43. Радченко В. П., Кичаев Е. К., Симонов А. В. Энергетический вариант модели реологического деформирования и разрушения металлов при совместном действии статических и циклических нагрузок // ПМТФ, 2000. Т. 41, № 3. С. 169-175.

44. Zheng X.-T., Xuan F.-Z., Zhao P. Ratcheting-creep interaction of advanced 9-12\% chromium ferrite steel with anelastic effect// Int. J. Fatigue, 2011. vol.33, no. 9. pp. 12861291. doi: 10.1016/j.ijfatigue.2011.04.009.

45. Баренблатт Г. И., Козырев Ю. И., Малинин Н. Н., Павлов Д. Я., Шестериков С. А. О виброползучести полимерных материалов // ПМТФ, 1965. № 5. С. 68-75.

46. Локощенко А. М., Шестериков С. А. О виброползучести // Инженерный журнал. Механика твердого тела, 1966. № 3. С. 141-143.

47. Локощенко А. М. Виброползучесть металлов при одноосном и сложном напряженных состояниях // Изв. РАН. МТT, 2014. № 4. С. 111-120.

48. Хохлов А. В. Характерные особенности семейств кривых деформирования линейных моделей вязкоупругости // Проблемы прочности и пластичности, 2015. Т. 77, № 2 . C. $139-154$.

49. Хохлов А. В. Кривые длительной прочности, порождаемые линейной теорией вязкоупругости в сочетании с критериями разрушения, учитывающими историю деформирования // Труды МАИ, 2016. №91. С. 1-32, http://www.mai.ru/science/trudy/ published. php?ID=75559.

50. Хохлов А. В. Анализ общих свойств кривых ползучести при ступенчатом нагружении, порождаемых нелинейным соотношением Работнова для вязкоупругопластичных материалов // Вестник МГТУ им. Н. Э. Баумана. Сер. Естественные науки, 2017. № 3. C. 93-123. doi : 10.18698/1812-3368-2017-3-93-123.

51. Радченко В. П., Самарин Ю. П. Влияние ползучести на величину упругой деформации слоистого композита // Механика композитных материалов, 1983. Т. 19, № 2. С. 231237.

52. Радченко В. П., Шапиевский Д. В. О дрейфе упругой деформации для нелинейноупругих материалов вследствие ползучести // Вестн. Сам. гос. техн. ун-та. Сер. Физ.-мат. науки, 2006. №43. С. 99-106. doi: 10.14498/vsgtu458.

53. Радченко В. П., Шапиевский Д. В. Математическая модель ползучести микронеоднородного нелинейно-упругого материала // ПМТФ, 2008. Т. 49, № 3. С. 157-163.

54. Мелнис А. Э., Лайзан Я. Б. Нелинейная ползучесть компактной костной ткани человека при растяжении // Механика полимеров, 1978. Т. 14, №1. С. 97-100. 


\title{
MSC: 74D05
}

\section{Analysis of creep curves produced by the linear viscoelasticity theory under cyclic stepwise loadings}

\section{A. V. Khokhlov}

Lomonosov Moscow State University, Institute of Mechanics,

1, Michurinsky prospekt, Moscow, 119192, Russian Federation.

\begin{abstract}
Basic qualitative properties of the creep curves generated by the linear integral constitutive relation of viscoelasticity (with an arbitrary creep compliance) under cyclic piecewise-constant uni-axial loadings (with an arbitrary asymmetry stress ratio) are studied analytically. General formulas and a number of exact two-sided bounds are obtained for maximal, minimal and ratcheting strain values during each cycle, for their sequences limits, for the rate of plastic (non-recoverable) strain accumulation and for cyclic creep curve deviation from the creep curve at constant stress which is equal to the cycle mean stress. Their dependence on loading cycle parameters and creep compliance properties are analyzed. Monotonicity and convexity intervals of cyclic creep curves, sequences of maximal and minimal strain values and ratcheting strain sequence, their evolution with cycle number growth and conditions for their boundedness, monotonicity and convergence are examined. The linear viscoelasticity theory abilities for simulation of ratcheting, creep acceleration, cyclic hardening or softening and cyclic stability under symmetric cyclic loadings are considered.

The analysis carried out revealed the importance of convexity restriction imposed on a creep compliance and the governing role of its derivative limit value at infinity. It is proved that the limit value equality to zero is the criterion for non-accumulation of plastic strain, for memory fading and for asymptotic symmetrization of cyclic creep curve deviation from the creep curve at the mean stress.

The qualitative features of theoretic cyclic creep curves are compared to basic properties of typical test creep curves of viscoelastoplastic materials under cyclic multi-step uni-axial loadings in order to elucidate the linear theory applicability scope, to reveal its abilities to provide an adequate description of basic rheological phenomena related to cyclic creep and to develop techniques of identification and tuning of the linear constitutive relation. In particular, it is proved that the linear constitutive relation with an arbitrary (increasing convex-up) creep compliance function provides the absence of ratcheting and cyclic softening under symmetric cyclic multi-step loadings and the absence of creep acceleration whenever a symmetric cyclic loading is added to a constant load.
\end{abstract}

\footnotetext{
Article ternational License (http://creativecommons.org/licenses/by/4.0/)

Please cite this article in press as: doi: 10.14498/vsgtu1533 (In Russian).

Author's Details:

Andrew V. Khokhlov (iD http://orcid.org/0000-0002-9212-2579

Cand. Techn. Sci.; Senior Researcher; Lab. of Elasticity and Plasticity;

e-mail: andrey-khokhlov@ya.ru
}

(우(i) The content is published under the terms of the Creative Commons Attribution 4.0 In-

Khokhlov A. V. Analysis of creep curves produced by the linear viscoelasticity theory under cyclic stepwise loadings, Vestn. Samar. Gos. Tekhn. Univ., Ser. Fiz.-Mat. Nauki [J. Samara State Tech. Univ., Ser. Phys. Math. Sci.], 2017, vol. 21, no. 2, pp. 326-361. 
Keywords: linear viscoelasticity, cyclic creep, creep curves at piecewiseconstant loading, asymmetry stress ratio, mean stress, creep acceleration, plastic strain, ratcheting, cyclic stability.

Received: $14^{\text {th }}$ March, $2017 /$ Revised: $17^{\text {th }}$ May, $2017 /$

Accepted: $12^{\text {th }}$ June, $2017 /$ First online: $10^{\text {th }}$ July, 2017

Competing interests. I have no competing interests.

Author's Responsibilities. I take full responsibility for submitting the final manuscript in print. I approved the final version of the manuscript.

Funding. This work was supported by the Russian Foundation for Basic Research (project no. 17-08-01146_a).

\section{References}

1. Kachanov L. M. Creep Theory. Moscow, Fizmatgiz, 1960, 456 pp. (In Russian)

2. Namestnikov B. C., Khvostunkov A. A. Creep of duralumin at constant and variable loads, Prikl. Mekh. Tekh. Fiz, 1960, vol. 1, no. 4, pp. 90-95 (In Russian).

3. Kennedy A. J. Processes of creep and fatigue in metals, Wiley series on the science and technology of materials, vol. 19. New York, Wiley, 1963, $480 \mathrm{pp}$.

4. Rabotnov Yu. N. Polzuchest' elementov konstruktsii [Creep of Structure Elements]. Moscow, Nauka, 1966, 752 pp. (In Russian)

5. Odqvist F. K. G. Mathematical Theory of Creep and Creep Rupture. Oxford, Clarendon ress, 1966, $170 \mathrm{pp}$.

6. Samarin Yu. P., Sorokin O. V. On the creep of masticated polyvinyl chloride rubber under variable loads, Doklady Akad. Nauk SSSR, 1970, vol. 195, no. 2, pp. 333-336 (In Russian).

7. Bugakov I. I. Polzuchest' polimernykh materialov [Creep of polymer materials]. Moscow, Nauka, 1973, 287 pp. (In Russian)

8. Findley W. N., Lai J. S., Onaran K. Creep and Relaxation of Nonlinear Viscoelastic aterials. Amsterdam, North Holland, 1976, 368 pp.

9. Strizhalo V. A. Tsiklicheskaia prochnost' $i$ polzuchest' metallov pri malotsiklovom nagruzhenii $v$ usloviiakh nizkikh i vysokikh temperatur [Cyclic Strength and Creep of Metals under Low-Cycle Loading at Low and High Temperatures]. Kiev, Naukova Dumka, 1978, 238 pp. (In Russian)

10. Kujawski D., Kallianpur V., Krempl E. An experimental study of uniaxial creep, cyclic creep and relaxation of aisi type 304 stainless steel at room temperature, J. Mech. Phys. Solids, 1980, vol. 28, no. 2, pp. 129-148. doi : 10.1016/0022-5096(80)90018-6.

11. Shesterikov S. A., Lokoshchenko A. M. Creep and Long-Term Strength of Metals, Itogi nauki i tekhniki. Ser. Mekhan. deform. tverd. tela [Results of Science and Engineering, Ser. Mechanics of Deformable Solids], 13. Moscow, VINITI, 1980, pp. 3-104 (In Russian).

12. Malinin N. N. Raschety na polzuchest' elementov mashinostroitel'nykh konstruktsii [Creep Calculations on Parts of Machine Constructions]. Moscow, Mashinostroenie, 1981, 221 pp. (In Russian)

13. Moskvitin V. V. Tsiklicheskoe nagruzhenie elementov konstruktsii [Cyclic Loading of Structure Elements]. Moscow, Nauka, 1981, 344 pp. (In Russian)

14. Lokoshchenko A. M., Namestnikova I. V., Shesterikov S. A. Description of longterm strength with stepwise stress changes, Strength of Materials, 1981, vol.13, no.10, pp. 1240-1244. doi : 10.1007/BF00775885.

15. Cho U. W., Findley W. N. Creep and Plastic Strains of 304 Stainless Steel at $593^{\circ} \mathrm{C}$ Under Step Stress Changes, Considering Aging, J. Appl. Mech., 1982, vol.49, no. 2, pp. 297-304. doi : 10.1115/1.3162084.

16. Golub V. P. Tsiklicheskaia polzuchest' zharoprochnykh nikelevykh splavov [Cyclic Creep of Creep-Resisting Nickel Alloys]. Kiev, Naumova dumka, 1983, 224 pp. (In Russian) 
17. Gokhfel'd D. A., Sadakov O. S. Plastichnost' i polzuchest' elementov konstruktsii pri povtornykh nagruzheniiakh [Plasticity and creep in structural elements under repeated loading]. Moscow, Mashinostroenie, 1984, 256 pp. (In Russian)

18. Malinin H N. Polzuchest' $v$ obrabotke metallov davleniem [Creep theories in metal forming]. Moscow, Mashinostroenie, 1986, 221 pp. (In Russian)

19. Golub V. P. Investigations into cyclic creep of materials (review), Soviet Applied Mechanics, 1987, vol. 23, no. 12, pp. 1107-1121. doi: 10.1007/BF00884887.

20. Tschoegl N. W. The Phenomenological Theory of Linear Viscoelastic Behavior. Berlin, Springer, 1989, 769 pp.

21. Nikitenko A. F. Polzuchest' i dlitel'naia prochnost' metallicheskikh materialov [Creep and Long-Time Strength of Metallic Materials]. Novosibirsk, Novosibirsk State Architectural Univ., 1997, 278 pp. (In Russian)

22. Lokoshchenko A. M. Polzuchest' $i$ dlitel'naia prochnost' metallov v agressivnykh sredakh [Creep and Long-Term Strength of Metals in Corrosive Media]. Moscow, Moscow State Univ., 2000, 179 pp. (In Russian)

23. Radchenko V. P., Saushkin M. N. Polzuchest' i relaksatsiia ostatochnykh napriazhenii v uprochnennykh konstruktsiiakh [Creep and Relaxation of Residual Stresses in Hardened Structures]. Moscow, Mashinostroenie-1, 2005, 226 pp. (In Russian)

24. Hamouda B. H., Laiarinandrasana L., Piques R. Viscoplastic behavior of a medium density polyethylene (MDPE): constitutive equations based on double nonlinear deformation model, Int. J. Plasticity, 2007, vol. 23, no. 8, pp. 1307-1327. doi: 10.1016/j.ijplas.2006.11.007.

25. Betten J. Creep Mechanics. Berlin, Heidelberg, Springer-Verlag, 2008, 267 pp.

26. Radchenko V. P., Kichaev P. E. Energeticheskaia kontseptsiia polzuchesti i vibropolzuchesti metallov [Energy Concept of Creep and Vibrocreep of Metals]. Samara, Samara State Techn. Univ., 2011, 157 pp. (In Russian)

27. Darabi M. K, Al-Rub R. K. A., Masad E. A., Huang C.-W., Little D. N. A modified viscoplastic model to predict the permanent deformation of asphaltic materials under cycliccompression loading at high temperatures, Int. J. Plasticity, 2012, vol.35, pp. 100-134. doi: 10.1016/j.ijplas.2012.03.001.

28. Bergstrom J. S. Mechanics of Solid Polymers. Theory and Computational Modeling. Elsevier, William Andrew, 2015, 520 pp.

29. Lokoshchenko A. M. Polzuchest' $i$ dlitel'naia prochnost' metallov [Creep and long-lasting strength of metals]. Moscow, Fizmatlit, 2016, 504 pp. (In Russian)

30. Lokoshchenko A. M., Fomin L. V. Creep fracture of plates with variable bending moments in the presence of an aggressive medium, J. Appl. Math. Mech., 2016, vol. 80, no. 2, pp. 198204. doi: 10.1016/j.jappmathmech.2016.06.006.

31. Dandrea J., Lakes R. S. Creep and creep recovery of cast aluminum alloys, Mech. TimeDepend. Mater., 2009, vol.13, no.4, pp. 303-315. doi: 10.1007/s11043-009-9089-6.

32. Taleb L., Cailletaud G. Cyclic accumulation of the inelastic strain in the 304L SS under stress control at room temperature: Ratcheting or creep?, Int. J. Plasticity, 2011, vol. 27, no. 12, pp. 1936-1958. doi: 10.1016/j.ijplas.2011.02.001.

33. Khan F., Yeakle C. Experimental investigation and modeling of non-monotonic creep behavior in polymers, Int. J. Plasticity, 2011, vol.27, no.4, pp. 512-521. doi: 10.1016/j. ijplas.2010.06.007.

34. Drozdov A. D. Time-dependent response of polypropylene after strain reversal, Int. J. of Solids and Structures, 2010, vol. 47, no. 24, pp. 3221-3233. doi : 10.1016/j . i jsolstr. 2010. 08.001.

35. Drozdov A. D., Dusunceli N. Unusual mechanical response of carbon black-filled thermoplastic elastomers, Mechanics of Materials, 2014, vol.69, no. 1, pp. 116-131. doi: 10.1016/ j.mechmat . 2013.09.019.

36. Khohlov A.V. Properties of creep curves at piecewise-constant stress generated by the linear viscoelasticity theory, Problemy prochnosti $i$ plastichnosti [Problems of strength and plasticity], 2015, vol. 77, no. 4, pp. 344-359 (In Russian). 
37. Khohlov A.V. The Qualitative Analysis of Theoretic Curves Generated by Linear Viscoelasticity Constitutive Equation, Science and Education, 2016, no. 5, pp. 187-245 (In Russian), http://technomag.bmstu.ru/doc/840650.html.

38. Khokhlov A. V. The nonlinear Maxwell-type viscoelastoplastic model: properties of creep curves at piecewise-constant stress and criterion for plastic strain accumulation, Mashinostroenie $i$ inzhenernoe obrazovanie, 2016, no. 3, pp. 55-68 (In Russian).

39. Khokhlov A. V. Asymptotic commutativity of creep curves at piecewise-constant stress produced by the linear viscoelasticity theory, Mashinostroenie $i$ inzhenernoe obrazovanie, 2016, no. 1, pp. 70-82 (In Russian).

40. Khokhlov A. V. Long-term strength curves generated by the nonlinear Maxwell-type model for viscoelastoplastic materials and the linear damage rule under step loading, Vestn. Samar. Gos. Tekhn. Univ., Ser. Fiz.-Mat. Nauki [J. Samara State Tech. Univ., Ser. Phys. Math. Sci.], 2016, vol. 20, no. 3, pp. 524-543 (In Russian). doi: 10.14498/vsgtu1512.

41. Fatemi A., Yang L. Cumulative fatigue damage and life prediction theories: A survey of the state of the art for homogeneous materials, Int. J. Fatigue, 1998, vol.20, no. 1, pp. 9-34. doi : 10.1016/S0142-1123(97)00081-9.

42. Golub V. P. Some creep effects in cyclic loading, Strength of Materials, 1987, vol. 19, no. 5, pp. 605-610. doi: 10.1007/bf01524289.

43. Radchenko V. P., Kichaev E. K., Simonov A. V. Energetic version of the model of rheological deformation and destruction of metal under a joint action of static and cyclic loads, J. Appl. Mech. Tech. Phys., 2000, vol.41, no. 3, pp. 531-537. doi: 10.1007/BF02465307.

44. Zheng X.-T., Xuan F.-Z., Zhao P. Ratcheting-creep interaction of advanced 9-12\% chromium ferrite steel with anelastic effect, Int. J. Fatigue, 2011, vol. 33, no. 9, pp. 12861291. doi: 10.1016/j.ijfatigue.2011.04.009.

45. Barenblatt G. I., Kozyrev Iu. I., Malinin N. N., Pavlov D. Ia., Shesterikov S. A. On the vibrocreep of polymeric materials, Prikl. Mekh. Tekh. Fiz., 1965, no. 5, pp. 68-75 (In Russian).

46. Lokoshchenko A. M., Shesterikov S. A. On Vibrocreep, Inzhenernyi zhurnal. Mekhanika tverdogo tela, 1966, no. 3, pp. 141-143 (In Russian).

47. Lokoshchenko A. M. Vibrocreep of metals in uniaxial and complex stress states, Mechanics of Solids, 2014, vol.49, no.4, pp. 453-460. doi: 10.3103/S0025654414040104.

48. Khokhlov A. V. Specific features of stress-strain curves at constant stress rate or strain rate yielding from linear viscoelasticity, Problemy prochnosti i plastichnosti [Problems of strength and plasticity], 2015, vol.77, no. 2, pp. 139-154 (In Russian).

49. Khokhlov A. V. Long-term strength curves produced by linear viscoelasticity theory combined with failure criteria accounting for strain history, Trudy MAI, 2016, no. 91, pp. 1-32 (In Russian), http://www .mai.ru/science/trudy/published.php?ID=75559.

50. Khokhlov A. V. Khokhlov A.V. Analysis of general properties of creep curves generated by the Rabotnov nonlinear hereditary relation under multi-step loadings, Vestn. Mosk. Gos. Tekh. Univ. im. N. E. Baumana, Estestv. Nauki [Herald of the Bauman Moscow State Technical University. Series Natural Sciences], 2017, no. 3, pp. 93-123 (In Russian). doi : 10.18698/1812-3368-2017-3-93-123.

51. Radchenko V. P., Samarin Yu. P. Effect of creep on the elastic deformation of a laminar composite, Mechanics of Composite Materials, 1983, vol.19, no. 2, pp. 162-168. doi: 10. 1007/BF00604218.

52. Radchenko V. P., Shapievskii D. V. Drift of elastic deformation due to creep for nonlinear elastic materials, Vestn. Samar. Gos. Tekhn. Univ., Ser. Fiz.-Mat. Nauki [J. Samara State Tech. Univ., Ser. Phys. Math. Sci.], 2006, no.43, pp. 99-106 (In Russian). doi: 10.14498/ vsgtu 458.

53. Radchenko V. P., Shapievskii D. V. Mathematical model of creep for a microinhomogeneous nonlinearly elastic material, J. Appl. Mech. Tech. Phys., 2008, vol.49, no. 3, pp. 478-483. doi: 10.1007/s10808-008-0064-9.

54. Melnis A. É., Laizan Ya. B. Nonlinear creep of human compact bone tissue upon stretching, Polymer Mechanics, 1978, vol. 14, no. 1, pp. 82-84. doi: 10.1007/BF00859565. 